\title{
APPROXIMATING GEODESICS VIA RANDOM POINTS ${ }^{1}$
}

\author{
By ERIK DAVIS AND Sunder SeThuraman
}

\section{University of Arizona}

\begin{abstract}
Given a cost functional $F$ on paths $\gamma$ in a domain $D \subset \mathbb{R}^{d}$, in the form $F(\gamma)=\int_{0}^{1} f(\gamma(t), \dot{\gamma}(t)) d t$, it is of interest to approximate its minimum cost and geodesic paths. Let $X_{1}, \ldots, X_{n}$ be points drawn independently from $D$ according to a distribution with a density. Form a random geometric graph on the points where $X_{i}$ and $X_{j}$ are connected when $0<\left|X_{i}-X_{j}\right|<\varepsilon$, and the length scale $\varepsilon=\varepsilon_{n}$ vanishes at a suitable rate.

For a general class of functionals $F$, associated to Finsler and other distances on $D$, using a probabilistic form of Gamma convergence, we show that the minimum costs and geodesic paths, with respect to types of approximating discrete cost functionals, built from the random geometric graph, converge almost surely in various senses to those corresponding to the continuum cost $F$, as the number of sample points diverges. In particular, the geodesic path convergence shown appears to be among the first results of its kind.
\end{abstract}

1. Introduction. Understanding the shortest or geodesic paths between points in a medium is an intrinsic concern in diverse applied problems, from optimal routing in networks and disordered materials to identifying manifold structure in large data sets, as well as in studies of $\mathbb{Z}^{d}$ and $\mathbb{R}^{d}$-percolation (cf. recent survey [5] and [17-22]).

There are sometimes abstract formulas for the geodesics, from the calculus of variations, or other differential equation approaches. For instance, with respect to a patch of a Riemannian manifold $(M, g)$, with $M \subset \mathbb{R}^{d}$ and tensor field $g(\cdot)$, it is known that the distance function $U(\cdot)=d(x, \cdot)$, for fixed $x$, is a viscosity solution of the Eikonal equation $\|\nabla U(y)\|_{g(y)^{-1}}=1$ for $y \neq x$, with boundary condition $U(x)=0$. Here, $\|v\|_{A}=\sqrt{\langle v, A v\rangle}$, where $\langle\cdot, \cdot\rangle$ is the standard inner product on $\mathbb{R}^{d}$. Then a geodesic $\gamma$ connecting $x$ and $z$ may be recovered from $U$ by solving a descent equation, $\dot{\gamma}(t)=-\eta(t) g^{-1}(\gamma(t)) \nabla U(\gamma(t))$, where $\eta(t)$ is a scalar function controlling the speed.

On the other hand, computing numerically the distances and geodesics may be a complicated issue. One of the standard approaches is the fast marching method to

Received December 2017; revised April 2018.

${ }^{1}$ Supported in part by ARO W911NF-14-1-0179, ARO W911NF-18-1-0311, and a Simons sabbatical grant.

MSC2010 subject classifications. Primary 60D05; secondary 58E10, 62-07, 49J55, 49J45, $53 \mathrm{C} 22,05 \mathrm{C} 82$.

Key words and phrases. Geodesic, shortest path, distance, consistency, random geometric graph, Gamma convergence, scaling limit, Finsler. 
approximate the distance $U$, by solving the Eikonal equation on a regular grid of $n$ points. This method has been extended in a variety of ways, including with respect to triangulated domains, as well as irregular samples $\left\{x_{1}, \ldots, x_{n}\right\}$ of an Euclidean submanifold (cf. [24, 30]). See also [27] in the above contexts for a review.

Alternatively, variants of Dijkstra's or heat flow methods, on graphs approximating the space are sometimes used. In Dijkstra's algorithm, distances and shortest paths are found by successively computing optimal routes to nearest-neighbor edges. In heat flow methods, geodesic distances can be found in terms of the small time asymptotics of a heat kernel on the space. For instance, see $[10,11,14,33$, 34].

Another idea has been to collect a random sample $\mathcal{X}_{n}$ of $n$ points from a manifold embedded in $\mathbb{R}^{d}$, put a network structure on these points, say in terms of a $\varepsilon$-random geometric or $k$-nearest neighbor graph, and then approximate the continuum geodesics lengths by lengths of discrete geodesic paths found in this network. Presumably, under assumptions on how the points are sampled and how the random graphs are formed, as the number of points diverge, these discrete distances should converge almost surely to the continuum shortest path lengths. Such a statistical consistency result is fundamental in manifold learning [6]. For instance, the popular ISOMAP procedure $[7,32]$ is based on these notions to elicit manifold structure in data sets.

More specifically, let $D$ be a subset of $\mathbb{R}^{d}$ corresponding to a patch of the manifold, and consider a kernel $f(x, v): D \times \mathbb{R}^{d} \rightarrow[0, \infty)$. Define the $f$-cost of a path $\gamma(t):[0,1] \rightarrow D$ from $\gamma(0)=a$ to $\gamma(1)=b$ as $F(\gamma)=\int_{0}^{1} f(\gamma(t), \dot{\gamma}(t)) d t$. The $f$-distance from $a$ to $b$ is then the infimum of such costs over paths $\gamma$. For example, if $f(x, v)=|v|^{p}$ and $p \geq 1$, the $f$-distance is $|b-a|^{p}$, the $p$ th power of the Euclidean distance. Moreover, when $p=1, F(\gamma)$ is the arc length of the path $\gamma$ and the $f$-distance is the length of the line segment from $a$ to $b$.

With respect to a class of functions $f$ and samples drawn from a distribution on the $D$ with density $\rho$, papers [7, 28] and [3] address, among other results, how $\varepsilon=\varepsilon_{n}$ and $k=k_{n}$ should decrease and increase, respectively, so that various concentration type bounds between types of discrete and continuum optimal distances hold with high probability, leading to consistent estimates.

For instance, in [28], for $\varepsilon_{n}$-random graphs and smooth $\rho$, certain density dependent estimators of continuum distances were considered, where $f(x, v)=$ $h(\rho(x))|v|$ and $h(y)$ is decreasing, smooth, constant for $|y|$ small, and bounded away from 0 . This work extends [7], which considered $f(x, v)=|v|$ and uniformly distributed samples. On the other hand, in [3], among other results, on $k_{n^{-}}$ nearest-neighbor graphs, continuum distances, where $f(x, v)=h(\rho(x))|v|$ and $h$ is increasing, Lipschitz, and bounded away from 0, were approximated (see also [15]).

In these contexts, the purpose of this article is twofold. First, we identify a general class of $f$-distances for which different associated discrete distances, formed from random $\varepsilon_{n}$-random geometric graphs on a domain $D \subset \mathbb{R}^{d}$, converge almost 
surely to them. Second, we describe when the associated discrete geodesic paths converge almost surely, in uniform and Hausdorff norms, to continuum $f$-distance geodesic paths, a type of consistency which appears to be among the first contributions of this kind. The main results are Theorems 2.1, 2.2, 2.4, 2.5, 2.6 and Corollary 2.3. See Section 2 and Section 2.3 for precise statements and related remarks.

We consider the following three different discrete costs. The first, $d_{1}$, optimizes on paths $\gamma$, starting and ending at $a$ and $b$, respectively, linearly interpolated between points in $\mathcal{X}_{n} \cup\{a, b\}$, where consecutive points are within $\varepsilon_{n}$ of each other, and the time to traverse each link is the same. The second, $d_{2}$, optimizes with respect to quasinormal interpolations between the points, using however the $f$ geodesic paths. The third, $d_{3}$, does not interpolate at all, and optimizes a "Riemann sum" cost $\frac{1}{m} \sum_{i=0}^{m-1} f\left(v_{i}, m\left(v_{i+1}-v_{i}\right)\right)$ where $m$ is the number of edges in the discrete path $\left\{v_{0}, \ldots, v_{m}\right\} \subset \mathcal{X}_{n} \cup\{a, b\}$. We note, discrete distances $d_{2}$ and $d_{3}$, in the setting $f(x, v)=|v|$ were introduced in [7], and density dependent versions were used in the results in [28]. The discrete distance $d_{1}$, although natural, seems not well considered in the literature.

The conditions we impose on $f$ include $p$-homogeneity in $v$ for $p \geq 1$, convexity and an ellipticity condition with respect to $v$, and a smoothness assumption away from $v \neq 0$. Such conditions include a large class of kernels $f$ associated to Finsler spaces, as well as those kernels considered in [28] and [7], with respect to $\varepsilon_{n}$-random graphs. The domain $D \subset \mathbb{R}^{d}$ is assumed to be bounded and convex. Also, we assume that the rate of decrease of $\varepsilon_{n}$ is such that the graph on $\mathcal{X}_{n}$ is connected for all large $n$. Moreover, this choice of $\varepsilon_{n}$ implies that the density of nearby points increases sufficiently quickly so that zig-zags in the optimal path approximations do not accumulate.

While a main contribution of the article is to provide a general setting in which the discrete to continuum convergences hold, we remark our proof method is quite different from that in the literature, where specific features of $f$, such as $f(x, v)=$ $|v|$ in [7], are important in estimation of distances, not easily generalized. We use a form of Gamma convergence to derive the almost sure limits, which may be of interest itself. This method involves showing liminf, limsup and compactness elements, as in the analysis context, but here on appropriate probability 1 sets. Part of the output of the technique, beyond giving convergence of the distances, is that it yields convergence of the minimizing discrete paths to continuum geodesics in various senses.

The $f$-costs share different properties depending on if $p=1$ or $p>1$, and also when $d \geq 2$ or $d=1$. For instance, the $f$-cost is invariant to reparametrization of the path exactly when $p=1$. Also, when $p>1$, the form of the $f$-cost may be seen to be coercive on the modulus of $\gamma$, not the case when $p=1$. In fact, the $p=1$ case is the most troublesome, and more assumptions on $f$ and $\varepsilon_{n}$ are required in Theorems 2.5 and 2.6 to deal with the linear path cost $d_{1}$ and Riemann cost $d_{3}$, which are rougher than the quasinormal cost $d_{2}$. 
At the same time, in $d=1$, in contrast to $d \geq 2$, all paths lie in the interval $[a, b] \subset \mathbb{R}$. When also $p=1$, the problem is somewhat degenerate: By invariance to reparametrization, the costs $d_{1}$ and $d_{2}$ turn out to be nonrandom and to reduce to the integral $\int_{a}^{b} f(s, 1) d s$. Also, the cost $d_{3}$ is a Riemann sum which converges to this integral.

Finally, we comment on a difference in viewpoint with respect to results in continuum percolation. The Riemann sum cost considered here seems related to but is different than the cost optimized in the works [18, 19]. There, for $p>1$, one optimizes the cost of a path $\left\{w_{0}, \ldots, w_{m}\right\}$, along random points, from the origin 0 to $n x$, for $x \in \mathbb{R}^{d}$, given by $\sum_{i=0}^{m-1}\left|w_{i+1}-w_{i}\right|^{p}$, and infers a scaled distance $d(x)=c(d, p)|x|$, in law of large numbers scale $n$, where the proportionality constant $c(d, p)$ is not explicit. In contrast, however, in this article, given already an integral $f$-distance, the viewpoint is to optimize costs of paths of length order 1 (not $n$ as in [18, 19]), where the length scale between points is being scaled of order $\varepsilon_{n}$, and then to recover the $f$-distance in the limit. We note also another difference: When $f(x, v)=|v|^{p}$, as remarked above, the $f$-distance from the origin to $x$ is $|x|^{p}$, instead of $\sim|x|$ as in the continuum percolation studies.

In Section 2, the setting, assumptions and results are given with respect to three types of discrete costs. In Section 3, proofs of Theorems 2.1 and 2.2 and Corollary 2.3 on the interpolating costs are given. In Section 4, proofs of Theorems 2.4, 2.5 and 2.6, with respect to Riemann costs, and interpolating costs when $p=1$, are given. In the Appendix, some technical results, used in the course of the main proofs, are collected.

2. Setting and results. We now introduce the setting of the problem, and standing assumptions, which hold throughout the article.

For $d \geq 1$, we will be working on a subset $D \subset \mathbb{R}^{d}$,

which is the closure of an open, bounded, convex domain.

Therefore, $D$ is a Lipschitz domain (cf. Corollary 9.1.2 in [2], Section 1.1.8 in [23]).

Consider points $a, b \in D$ and let $\Omega(a, b)$ denote the space of Lipschitz paths $\gamma:[0,1] \rightarrow D$ with $\gamma(0)=a$ and $\gamma(1)=b$. Given $f: D \times \mathbb{R}^{d} \rightarrow[0, \infty)$, we define the cost $F: \Omega(a, b) \rightarrow[0, \infty)$ by

$$
F(\gamma)=\int_{0}^{1} f(\gamma(t), \dot{\gamma}(t)) d t
$$

and associated optimal cost

$$
d_{f}(a, b)=\inf _{\gamma \in \Omega(a, b)} F(\gamma) .
$$

We will make the following assumptions on the integrand $f$ :

(A0) $f$ is continuous on $D \times \mathbb{R}^{d}$, and $C^{1}$ on $D \times\left(\mathbb{R}^{d} \backslash\{0\}\right)$, 
(A1) $f(x, v)$ is convex in $v$,

(A2) there exists $p \geq 1$ such that $f(x, v)$ is $p$-homogenous in $v$,

$$
f(x, \lambda v)=\lambda^{p} f(x, v) \quad \text { for } \lambda>0,
$$

(A3) there exist constants $m_{1}, m_{2}>0$ such that

$$
m_{1}|v|^{p} \leq f(x, v) \leq m_{2}|v|^{p} \quad \text { for all } x \in D .
$$

We remark, when $p>1$ and $p$-homogeneity (A2) holds, that $f$ may be extended to a $C^{1}$ function on $D \times \mathbb{R}^{d}$.

Part of the reasoning for the assumptions (A0)-(A3) is that they include, for $p \geq$ 1 , the familiar kernel $f(x, v)=|v|^{p}$, mentioned in the Introduction, for which, when $p=1, F(\gamma)$ is the arclength of the path $\gamma$ and $d_{f}(a, b)=|b-a|$.

Also, under these assumptions on $f$, it is known that the infimum in (2.2) is attained at a path in $\Omega(a, b)$, perhaps nonuniquely (see Proposition A. 2 of the Appendix). In addition, we remark, when $p=1$, under additional differentiability assumptions, $d_{f}$ represents a Finsler distance (cf. [4, 31] and references therein).

When $p=1$, there is an interesting scaling property: By 1-homogeneity of $f$, the cost $F$ is invariant under smooth reparametrization of paths. That is, given a path $\gamma \in \Omega(a, b)$ and smooth, increasing $s:[0,1] \rightarrow[0,1]$, with $s(0)=0$ and $s(1)=1$, one has $F(\tilde{\gamma})=F(\gamma)$ where $\tilde{\gamma}(t)=\gamma(s(t))$.

This property allows to deduce, when $p=1$, that $d_{f}$ satisfies the triangle property (not guaranteed when $p>1$ ): Let $\gamma_{1}$ be a path from $u$ to $w$, and $\gamma_{2}$ be a path from $w$ to $z$.

Write

$$
\begin{aligned}
\int_{0}^{1} f & \left(\gamma_{1}(t), \dot{\gamma}_{1}(t)\right) d t+\int_{0}^{1} f\left(\gamma_{2}(t), \dot{\gamma}_{2}(t)\right) d t \\
& =\int_{0}^{1 / 2} f\left(\gamma_{1}(2 s), 2 \dot{\gamma}_{1}(2 s)\right) d s+\int_{0}^{1 / 2} f\left(\gamma_{2}(2 s), 2 \dot{\gamma}_{2}(2 s)\right) d s \\
& =\int_{0}^{1} f\left(\gamma_{3}(t), \dot{\gamma}_{3}(t)\right) d t
\end{aligned}
$$

where $\gamma_{3}$ is a path from $u$ to $z$, following $\gamma_{1}(2 \cdot)$ and $\gamma_{2}(2 \cdot)$ on time intervals $[0,1 / 2]$ and $[1 / 2,1]$, respectively. Optimizing over $\gamma_{1}, \gamma_{2}$ and $\gamma_{3}$ gives $d_{f}(u, w)+d_{f}(w, z) \geq d_{f}(u, z)$.

We now construct a random geometric graph on $D$ through which approximations of $d_{f}$ and its geodesics will be made. Let $\left\{X_{1}, X_{2}, \ldots\right\} \subset D$ be a sequence of independent points, identically distributed according to a distribution $v$ with probability density $\rho$. For each $n \in \mathbb{N}$, let $\mathcal{X}_{n}=\left\{X_{1}, \ldots, X_{n}\right\}$ and fix a length scale $\varepsilon_{n}>0$. With respect to a realization $\left\{X_{i}\right\}$, we define a graph $\mathcal{G}_{n}(a, b)$, on the vertex set $\mathcal{X}_{n} \cup\{a, b\}$, by connecting an edge between $u, v$ in $\mathcal{X}_{n} \cup\{a, b\}$ iff $0<|u-v|<\varepsilon_{n}$, where $|\cdot|$ refers to the Euclidean distance in $\mathbb{R}^{d}$. 


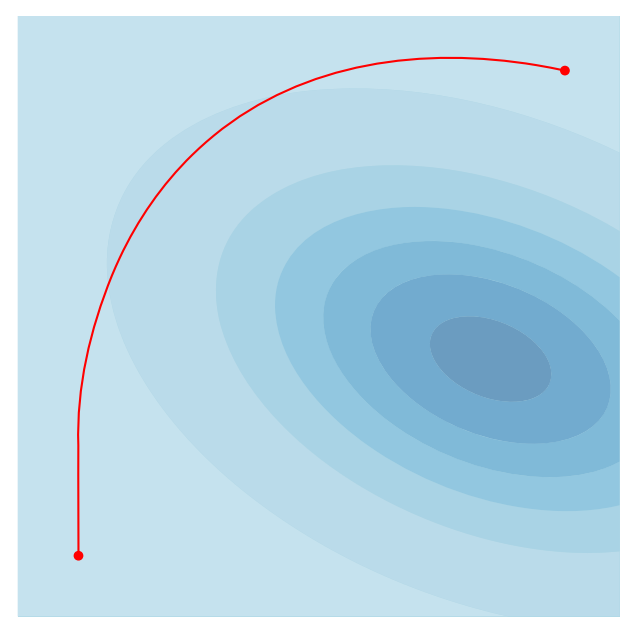

(a) F-minimizing path, with level sets of $w$ indicated.

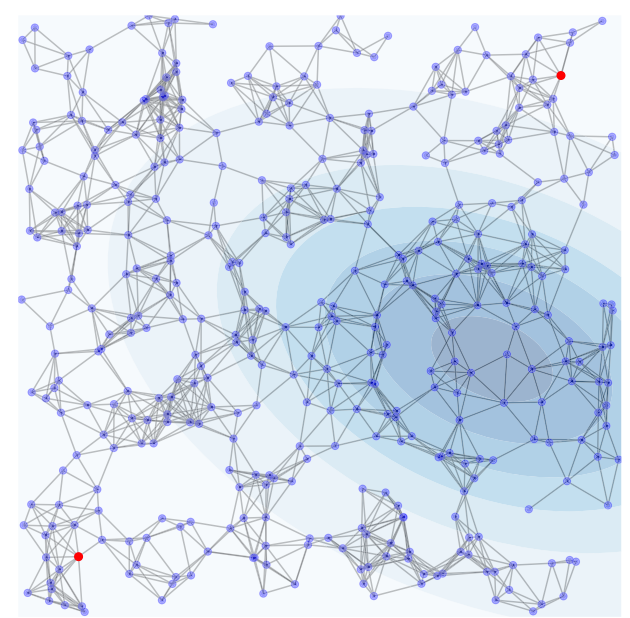

(b) $\epsilon_{n}$-graph, on $n=400$ uniform points with $\epsilon_{n}=(1 / 400)^{0.3}$

FIG. 1. Continuum geodesic and $\varepsilon_{n}$-graph for $f(x, v)=w(x)|v|$ on the domain $D=[-1,1] \times$ $[-1,1]$, where $w(x)=1+8 \exp \left(-2\left(x_{1}-1 / 2\right)^{2}+x_{1} x_{2}+2 x_{2}^{2}\right)$, and $a=(-0.8,-0.8), b=$ $(0.8,0.8)$.

For $u, v \in \mathcal{X}_{n} \cup\{a, b\}$, we say that a finite sequence $\left(v_{0}, v_{1}, \ldots, v_{m}\right)$ of vertices is a path with $m$-steps from $u$ to $v$ in $\mathcal{G}_{n}(a, b)$ if $v_{0}=u, v_{m}=v$, and there is an edge from $v_{i}$ to $v_{i+1}$ for $0 \leq i<m$. Let $V_{n}(a, b)$ denote the set of paths from $a$ to $b$ in $\mathcal{G}_{n}(a, b)$.

We will assume a certain decay rate on $\varepsilon_{n}$, namely that $\lim _{n \uparrow \infty} \varepsilon_{n}=0$ and

$$
\limsup _{n \rightarrow \infty} \frac{(\log n)^{1 / d}}{n^{1 / d}} \frac{1}{\varepsilon_{n}}=0 .
$$

Under this type of decay rate, almost surely, for all large $n$ and $a, b \in D$, points $a, b$ will be connected by a path in the graph $\mathcal{G}_{n}(a, b)$, in other words, $V_{n}(a, b)$ will be nonempty. Indeed, under this rate, the degree of a point in the graph will diverge to infinity. See Proposition A.1 in the Appendix, and remarks in Section 2.3.

We will also assume that the underlying probability density $\rho$ is uniformly bounded, that is, there exists a constant $c>0$ such that

$$
c \leq \rho(x) \leq c^{-1} \quad \text { for all } x \in D .
$$

See Figure 1, parts (a) and (b), which depict a geodesic path with respect to a cost $F$, and an $\varepsilon_{n}$-random graph.

Standing assumptions. To summarize, the assumptions, dimension $d \geq 1,(2.1)$ on $D$, items (A0)-(A3) on $f$ when $p \geq 1$, decay rate (2.6) on $\varepsilon_{n}$, and density bound (2.7) on $\rho$, denoted as the standing assumptions, will hold throughout the article. 
In the next two subsections, we present results on approximation of $d_{f}(a, b)$ and its geodesics with respect to two types of schemes, where approximating costs are built (1) in terms of interpolations of points in $V_{n}(a, b)$ and also (2) in terms of Riemann sums.

2.1. Interpolating costs. We introduce two types of discrete costs based on linear and quasinormal paths.

Linear interpolations. With respect to a realization $\left\{X_{i}\right\}$, for $u, v \in D$, let $l_{u, v}$ : $[0,1] \rightarrow D$ denote the constant-speed linear path from $a$ to $b$, given by

$$
l_{u, v}(t)=(1-t) u+t v .
$$

Consider now $\mathbf{v}=\left(v_{0}, v_{1}, \ldots, v_{m}\right) \in V_{n}(a, b)$. We define $l_{\mathbf{v}} \in \Omega(a, b)$ to be the concatenation of the linear segments $\left\{l_{v_{i-1}, v_{i}}\right\}_{i=1}^{m}$, where each segment is traversed in the same time $1 / m$. More precisely, for $i / m \leq t \leq(i+1) / m$, define

$$
l_{\mathbf{v}}(t)=l_{v_{i}, v_{i+1}}(m t-i),
$$

and note that the resulting piecewise linear path is in $\Omega(a, b)$.

Define now a subset $\Omega_{n}^{l}(a, b)$ of $\Omega(a, b)$ by

$$
\Omega_{n}^{l}(a, b)=\left\{l_{\mathbf{v}} \mid \mathbf{v} \in V_{n}(a, b)\right\},
$$

and define the (random) discrete cost $L_{n}: \Omega_{n}^{l}(a, b) \rightarrow[0, \infty]$ by

$$
L_{n}(\gamma)=F(\gamma) \quad \text { for } \gamma \in \Omega_{n}^{l}(a, b) .
$$

In other words, $L_{n}$ is the restriction of $F$ to $\Omega_{n}^{l}(a, b)$. By the $p$-homogeneity of $f$, we may write this as

$$
\begin{aligned}
L_{n}\left(l_{\mathbf{v}}\right) & =\sum_{i=0}^{m-1} \int_{i / m}^{(i+1) / m} f\left(l_{v_{i}, v_{i+1}}(m t-i), m\left(v_{i+1}-v_{i}\right)\right) d t \\
& =m^{p-1} \sum_{i=0}^{m-1} \int_{0}^{1} f\left(l_{v_{i}, v_{i+1}}(t), v_{i+1}-v_{i}\right) d t .
\end{aligned}
$$

Quasinormal interpolations. Define now a different discrete cost which may nonlinearly interpolate among points in paths of $V_{n}(a, b)$. We say that a Lipschitz path $\gamma$ is quasinormal with respect to $f$ if there exists a $c>0$ such that

$$
f(\gamma(t), \dot{\gamma}(t))=c \quad \text { for a.e. } t \in[0,1] .
$$

It is known, under the standard assumptions on $f$ (see Proposition A.2) that, for $u, v \in D$, there exists a quasinormal path $\gamma:[0,1] \rightarrow D$, with $\gamma(0)=u, \gamma(1)=v$, which is optimal, $d_{f}(u, v)=\int_{0}^{1} f(\gamma(t), \dot{\gamma}(t)) d t$. For what follows, when we refer to a quasinormal path connecting $u$ and $v$, we mean such a fixed optimal path denoted by $\gamma_{u, v}$. 
Given a path $\mathbf{v}=\left(v_{0}, \ldots, v_{m}\right) \in V_{n}(a, b)$, let $\gamma_{\mathbf{v}} \in \Omega(a, b)$ denote the concatenation of $\left\{\gamma_{v_{i-1}}, v_{i}\right\}_{i=1}^{m}$, where each segment uses the same time $1 / \mathrm{m}$. More precisely, for $i / m \leq t \leq(i+1) / m$, define

$$
\gamma_{\mathbf{v}}(t)=\gamma_{v_{i}, v_{i+1}}(m t-i) .
$$

As with piecewise linear functions, define the subset $\Omega_{n}^{\gamma}(a, b)$ of $\Omega(a, b)$ by

$$
\Omega_{n}^{\gamma}(a, b)=\left\{\gamma_{\mathbf{v}} \mid \mathbf{v} \in V_{n}(a, b)\right\} .
$$

Let $G_{n}: \Omega_{n}^{\gamma}(a, b) \rightarrow \mathbb{R}$ denote the restriction of $F$ to $\Omega_{n}^{\gamma}(a, b)$.

Then, with respect to a path $\gamma=\gamma_{\mathbf{v}} \in \Omega_{n}^{\gamma}(a, b)$, by the $p$-homogeneity of $f$, we evaluate that

$$
\begin{aligned}
G_{n}(\gamma) & =\int_{0}^{1} f(\gamma(t), \dot{\gamma}(t)) d t \\
& =\sum_{i=1}^{m} \int_{(i-1) / m}^{i / m} f\left(\gamma_{v_{i-1}, v_{i}}(m t-i), m \dot{\gamma}_{v_{i-1}, v_{i}}(m t-i)\right) d t \\
& =m^{p-1} \sum_{i=1}^{m} \int_{0}^{1} f\left(\gamma_{v_{i-1}, v_{i}}(t), \dot{\gamma}_{v_{i-1}, v_{i}}(t)\right) d t \\
& =m^{p-1} \sum_{i=1}^{m} d_{f}\left(v_{i-1}, v_{i}\right) .
\end{aligned}
$$

Further, by $p$-homogeneity of $f$ and optimality of $\left\{\gamma_{v_{i}, v_{i+1}}\right\}_{i=1}^{m}$, the segments of $\gamma=\gamma_{\mathbf{v}}$ are also optimal, in the sense that

$$
\begin{array}{rl}
\int_{i / m}^{(i+1) / m} & f(\gamma(t), \dot{\gamma}(t)) d t \\
\quad= & m^{p-1} \int_{0}^{1} f\left(\gamma_{v_{i}, v_{i+1}}(t), \dot{\gamma}_{v_{i}, v_{i+1}}(t)\right) d t \\
\quad & \inf _{\widetilde{\gamma}} m^{p-1} \int_{0}^{1} f(\widetilde{\gamma}(t), \dot{\tilde{\gamma}}(t)) d t=\inf _{\widehat{\gamma}} \int_{i / m}^{(i+1) / m} f(\widehat{\gamma}(t), \dot{\hat{\gamma}}(t)) d t,
\end{array}
$$

where the infima are over Lipschitz paths $\tilde{\gamma}:[0,1] \rightarrow D$ and $\widehat{\gamma}:[i / m,(i+$ $1) / m] \rightarrow D$ with $\widetilde{\gamma}(0)=v_{i}, \widetilde{\gamma}(1)=v_{i+1}, \widehat{\gamma}(i / m)=v_{i}$ and $\widehat{\gamma}((i+1) / m)=v_{i+1}$.

Relations between $G_{n}$ and $L_{n}$. At this point, we remark there are kernels $f$ for which $G_{n}=L_{n}$, namely those such that linear segments are in fact quasinormal geodesics. An example is $f(x, v)=|v|$. Identifying these kernels is a question with a long history, going back to Hilbert, whose fourth problem paraphrased asks for which geometries are the geodesics straight lines (cf. surveys [4, 25]). Hamel's criterion, namely $\partial_{x_{i}} \partial_{v_{j}} f=\partial_{x_{j}} \partial_{v_{i}} f$ for $1 \leq i, j \leq d$, is a well-known solution to this question (see $[4,13,25]$ and references therein). 
We also note, as mentioned in the Introduction, that the case $d=p=1$ is degenerate in that $\min G_{n}$ and $\min L_{n}$ are not random. Indeed, let $\gamma_{\mathbf{v}} \in \arg \min G_{n}$ and suppose $\mathbf{v}=\left(v_{0}, \ldots, v_{m}\right) \in V_{n}(a, b)$. We observe that $\gamma_{\mathbf{v}}$ must be nondecreasing, as otherwise, one could build a smaller cost path, from parts of $\gamma_{\mathbf{v}}$ using invariance to reparametrization, violating optimality of $\gamma_{\mathbf{v}}$. In particular, $\dot{\gamma}_{\mathbf{v}} \geq 0$ and $v_{i}<v_{i+1}$ for $0 \leq i \leq m-1$. Then

$$
\begin{aligned}
G_{n}\left(\gamma_{\mathbf{v}}\right) & =\sum_{i=0}^{m-1} \int_{i / m}^{(i+1) / m} f\left(\gamma_{\mathbf{v}}(t), \dot{\gamma}_{\mathbf{v}}(t)\right) d t \\
& =\sum_{i=0}^{m-1} \int_{v_{i}}^{v_{i+1}} f(s, 1) d s=\int_{a}^{b} f(s, 1) d s
\end{aligned}
$$

using the 1-homogeneity of $f$ and changing variables. The same argument yields that $\min L_{n}=\int_{a}^{b} f(s, 1) d s$. We do not consider this degenerate case further.

The first result is for linearly interpolated paths.

THEOREM 2.1. Suppose that $p>1$. With respect to realizations $\left\{X_{i}\right\}$ in a probability 1 set, the following holds. The minimum values of the costs $L_{n}$ converge to the minimum of $F$,

$$
\lim _{n \rightarrow \infty} \min _{\gamma \in \Omega_{n}^{l}(a, b)} L_{n}(\gamma)=\min _{\gamma \in \Omega(a, b)} F(\gamma) .
$$

Moreover, consider a sequence of optimal paths $\gamma_{n} \in \arg \min L_{n}$. Any subsequence of $\left\{\gamma_{n}\right\}$ has a further subsequence that converges uniformly to a limit path $\gamma \in \arg \min F$,

$$
\lim _{k \rightarrow \infty} \sup _{0 \leq t \leq 1}\left|\gamma_{n_{k}}(t)-\gamma(t)\right|=0 .
$$

In addition, if $\gamma$ is the unique minimizer of $F$, then the whole sequence $\gamma_{n}$ converges uniformly to $\gamma$.

The case $d \geq 2$ and $p=1$ requires further development, and is addressed with a few more assumptions in Theorem 2.6.

We now address quasinormal interpolations.

THEOREM 2.2. Suppose that either (1) $p>1$ or (2) $d \geq 2$ and $p=1$. Then, with respect to realizations $\left\{X_{i}\right\}$ in a probability 1 set, the following holds. The minimum values of the energies $G_{n}$ converge to the minimum of $F$,

$$
\lim _{n \rightarrow \infty} \min _{\gamma \in \Omega_{n}^{\gamma}(a, b)} G_{n}(\gamma)=\min _{\gamma \in \Omega(a, b)} F(\gamma) .
$$

Moreover, consider a sequence of optimal paths $\gamma_{n} \in \arg \min G_{n}$. Any subsequence of $\left\{\gamma_{n}\right\}$ has a further subsequence that converges uniformly to a limit path 
$\gamma \in \arg \min F$,

$$
\lim _{k \rightarrow \infty} \sup _{0 \leq t \leq 1}\left|\gamma_{n_{k}}(t)-\gamma(t)\right|=0 .
$$

In addition, if $\gamma$ is the unique minimizer of $F$, then the whole sequence $\gamma_{n}$ converges uniformly to $\gamma$.

We remark, when $d \geq 2$ and $p=1$, that there is a certain ambiguity in the results of Theorem 2.2, due to the invariance of $F$ under reparametrization of paths. In this case, there is no unique minimizer of $F$. Consider, for example, the case where $f(x, v)=|v|$ and $F(\gamma)=\int_{0}^{1}|\dot{\gamma}(t)| d t$. Any minimizer of this functional is a parametrization of a line, but of course such minimizers are not unique.

One way to address this is to formulate a certain Hausdorff convergence with respect to images of the paths. Given $\gamma \in \Omega(a, b)$, we denote the image of $\gamma$ by

$$
S_{\gamma}=\{\gamma(t) \mid 0 \leq t \leq 1\} \text {. }
$$

Consider the Hausdorff metric $d_{\text {haus }}$, defined on nonempty compact subsets $A, B$ of $D$ by

$$
d_{\text {haus }}(A, B)=\max \left\{\sup _{x \in A} \inf _{y \in B} d(x, y), \sup _{y \in B} \inf _{x \in A} d(x, y)\right\}
$$

COROllary 2.3. Suppose that either (1) $d \geq 2$ and $p=1$ or (2) $p>1$. Consider paths $\left\{\gamma_{\mathbf{v}^{(n)}}\right\}$, for all large $n$ either in form $\gamma_{\mathbf{v}^{(n)}} \in \arg \min G_{n}$, or $\gamma_{\mathbf{v}^{(n)}} \in$ $\arg \min L_{n}$.

Then, with respect to realizations $\left\{X_{i}\right\}$ in a probability 1 set, any subsequence of $\left\{\mathbf{v}^{(n)}\right\}$ has a further subsequence which converges in the Hausdorff sense to $S_{\gamma}$, where $\gamma \in \arg \min F$ is an optimal path.

Moreover, if $F$ has a unique (up to reparametrization) minimizer $\gamma$, then the whole sequence converges,

$$
\lim _{n \rightarrow \infty} d_{\text {haus }}\left(\mathbf{v}^{(n)}, S_{\gamma}\right)=0 .
$$

2.2. Riemann sum costs and $p=1$-linear interpolating costs. We first introduce a cost which requires knowledge of $f$ only on discrete points and, as a consequence, more applicable. At the end of the subsection, we return to linear interpolated costs when $p=1$.

Define $H_{n}: V_{n}(a, b) \rightarrow \mathbb{R}$, for $\mathbf{v}=\left(v_{0}, v_{1}, \ldots, v_{m}\right)$, by

$$
H_{n}(\mathbf{v})=\frac{1}{m} \sum_{i=1}^{m} f\left(v_{i}, m\left(v_{i+1}-v_{i}\right)\right) .
$$

The functional $H_{n}$ is, in a sense, a Riemann sum approximation to $L_{n}$ and $G_{n}$ and, therefore, its behavior, and the behavior of its minimizing paths, should be similar to that of $L_{n}$ and $G_{n}$. See Figure 2 for an example of an optimal $H_{n}$ path. 


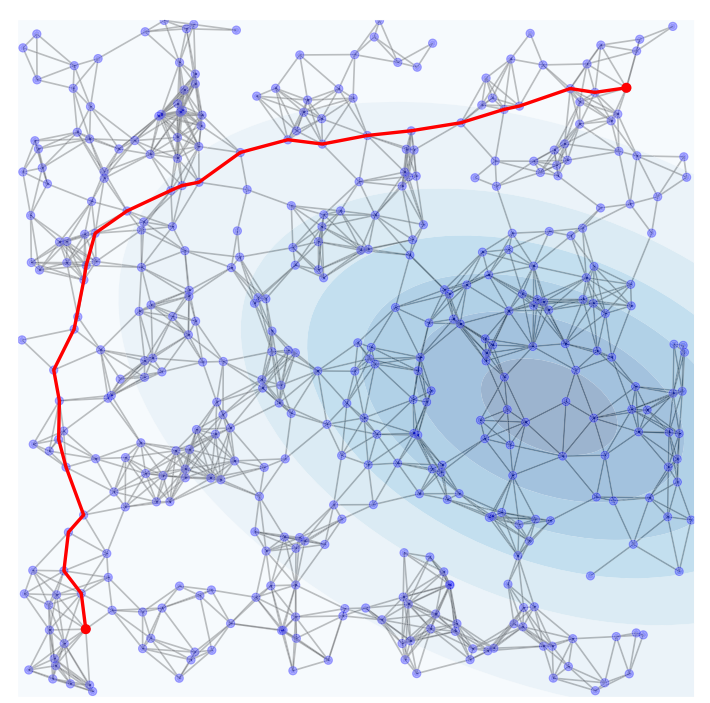

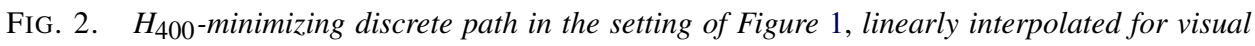
clarity (see Section 2.2).

We make this intuition rigorous by establishing variants of Theorems 2.1 and 2.2 with respect to the cost $H_{n}$. Given the rougher nature of $H_{n}$, however, additional assumptions on $f$ and $\varepsilon_{n}$, beyond those in the standing assumptions, will be helpful in this regard. As in the previous subsection, our results differ between the two cases $p=1$ and $p>1$.

Define the following smoothness condition:

(Lip) There exists a $c$ such that for all $x, y \in D$ and $v \in \mathbb{R}^{d}$ we have

$$
|f(x, v)-f(y, v)| \leq c|x-y||v|^{p} .
$$

We note when $f$ satisfies the homogeneity condition (2.3), and $\nabla_{x} f(x, v)$ is uniformly bounded on $D \times\{y:|y|=1\}$, that (Lip) holds.

We now consider the behavior of $H_{n}$ when $p>1$. The analogue to Theorem 2.1 and Corollary 2.3 in this setting is the following.

THEOREM 2.4. Suppose $p>1$, and that $f$ in addition satisfies (Lip). With respect to realizations $\left\{X_{i}\right\}$ in a probability 1 set, the minimum values of the energies $H_{n}$ converge to the minimum of $F$,

$$
\lim _{n \rightarrow \infty} \min _{\mathbf{v} \in V_{n}(a, b)} H_{n}(\mathbf{v})=\min _{\gamma \in \Omega(a, b)} F(\gamma) .
$$

Further, consider a sequence of optimal discrete paths $\mathbf{w}^{(n)} \in \arg \min H_{n}$, and their linear interpolations $\left\{l_{\mathbf{w}^{(n)}}\right\}$. Then, for any subsequence of $\left\{l_{\mathbf{w}^{(n)}}\right\}$ and correspondingly of $\left\{\mathbf{w}^{(n)}\right\}$, there is a further subsequence of the linear paths which 
converges uniformly to a limit path $\gamma \in \arg \min F$, and of the discrete paths in the Hausdorff sense to $S_{\gamma}$.

If $F$ has a unique minimizer $\gamma$, the whole sequence of linear paths converges uniformly to $\gamma$, and the whole sequence of discrete paths converges in the Hausdorff sense to $S_{\gamma}$.

We will need to impose further assumptions on the integrand $f$ to state results in the case $p=1$.

(Hilb) We say that $f$ satisfies the "Hilbert condition" if, for each $x$,

$$
\inf _{\gamma \in \Omega(a, b)} \int_{0}^{1} f(x, \dot{\gamma}(t)) d t=f(x, b-a),
$$

that is, straight lines are geodesics for the kernel $f(x, \cdot)$.

(TrIneq) We say $f$ satisfies the triangle inequality if, for each $x$,

$$
f(x, v-w) \leq f(x, v-u)+f(x, u-w)
$$

for all $u, v, w \in \mathbb{R}^{d}$.

(Pythag) Let $\alpha>1$. Consider points $u, v, w$ where $|u w|,|v w|,|u v|<\eta$ for a $\eta<1$. Suppose there is a constant $c$ such that, for $0<r<1$ :

$-\operatorname{dist}(w, \operatorname{line}(u, v)) \geq r$ and

$-|u v| \leq c r^{1 / \alpha}$.

Then we say $f$ satisfies the Pythagoras $\alpha$-condition if there is a constant $C=$ $C(\alpha, f, c)$ such that, for all $x$,

$$
f(x, w-u)+f(x, v-w) \geq f(x, v-u)+C r^{\alpha} .
$$

Here, line $(u, v)$ is the line segment between $u$ and $v$, and $|u v|=|u-v|$.

An example of a large class of kernels $f$, including $f(x, v)=|v|$, satisfying the standing assumptions and the additional conditions above is given in Section 2.4. See also Section 2.3 for further comments.

We will also need to limit the decay properties of $\varepsilon_{n}$ for the next result; see Section 2.3 for comments on this limitation. Namely, we will suppose that $\varepsilon_{n}$ is in form $\varepsilon_{n}=n^{-\delta}$ where

$$
\delta>\max \left\{\left[\left(2-\alpha^{2}\right) \eta+d\right]^{-1},\left[(\alpha(d-1)+1]^{-1}\right\},\right.
$$

for an $0<\eta<1$ and $1<\alpha<\sqrt{2}$.

We note that condition (2.6), when $\varepsilon_{n}$ is in form $\varepsilon_{n}=n^{-\delta}$, yields that $\delta<1 / d$. However, when $d \geq 2$, we have $\max \left\{\left[\left(2-\alpha^{2}\right) \eta+d\right]^{-1},\left[(\alpha(d-1)+1]^{-1}\right\}<1 / d\right.$, and so (2.12), in conjunction with (2.6), limits $\delta$ to an interval. 
THEOREM 2.5. Suppose $d \geq 2$ and $p=1$, and that $f$ also satisfies (Lip) and (Hilb). With respect to realizations $\left\{X_{n}\right\}_{n \geq 1}$ in a probability 1 set, the minimum values of the cost $H_{n}$ converge to the minimum of $F$,

$$
\lim _{n \rightarrow \infty} \min _{\mathbf{v} \in V_{n}(a, b)} H_{n}(\mathbf{v})=\min _{\gamma \in \Omega(a, b)} F(\gamma) .
$$

Moreover, suppose now that $f$ in addition satisfies (TrIneq) and (Pythag) for an $1<\alpha<\sqrt{2}$, and that $\varepsilon_{n}$ satisfies (2.12).

Consider a sequence of optimal discrete paths $\mathbf{v}^{(n)} \in \arg \min H_{n}$, and their linear interpolations $\left\{l_{\mathbf{v}^{(n)}}\right\}$. Then, for any subsequence of $\left\{l_{\mathbf{v}^{(n)}}\right\}$ and so of $\left\{\mathbf{v}^{(n)}\right\}$, there is a further subsequence of the linear paths which converges uniformly to a limit path $\gamma \in \arg \min F$, and of the discrete paths in the Hausdorff sense to $S_{\gamma}$.

If $F$ has a unique (up to reparametrization) minimizer $\gamma$, the whole sequence of discrete paths converges, $\lim _{n \rightarrow \infty} d_{\text {haus }}\left(\mathbf{v}^{(n)}, S_{\gamma}\right)=0$.

See Figure 2 for an example of an $H_{n}$-cost geodesic path.

As noted in the Introduction, when $d=p=1, H_{n}(\mathbf{v})$ is a certain Riemann sum. Let $\mathbf{w} \in \arg \min H_{n}$, and observe by optimality that $\mathbf{w}=\left(w_{0}, \ldots, w_{m}\right) \in$ $V_{n}(a, b)$ satisfies $w_{i}<w_{i+1}$ for $0 \leq i \leq m-1$. Hence, by 1-homogeneity of $f$, $H_{n}(\mathbf{w})=\sum_{i=0}^{m-1} f\left(w_{i}, 1\right)\left|w_{i+1}-w_{i}\right|$, and $H_{n}(\mathbf{w})$ strongly approximates the integral $\int_{a}^{b} f(s, 1) d s$, given that the partition length $\max \left|w_{i+1}-w_{i}\right| \leq \varepsilon_{n} \rightarrow 0$. For this reason, this case is not included in the above theorem.

Linear interpolating costs when $p=1$. Having now introduced (Lip), (Hilb), (TrIneq) and (Pythag), we address the case $d \geq 2$ and $p=1$ with respect to the $\operatorname{cost} L_{n}$.

THEOREM 2.6. Suppose $d \geq 2$ and $p=1$, and that $f$ also satisfies (Lip) and (Hilb). With respect to realizations $\left\{X_{n}\right\}_{n \geq 1}$ in a probability 1 set, the minimum values of the cost $L_{n}$ converge to the minimum of $F$,

$$
\lim _{n \rightarrow \infty} \min _{\gamma \in \Omega_{n}^{l}(a, b)} L_{n}(\gamma)=\min _{\gamma \in \Omega(a, b)} F(\gamma)
$$

Moreover, suppose now that $f$ in addition satisfies (TrIneq) and (Pythag) for an $1<\alpha<\sqrt{2}$, and that $\varepsilon_{n}$ satisfies (2.12).

Consider a sequence of optimal paths $l_{\mathbf{v}^{(n)}} \in \arg \min L_{n}$. Then, for any subsequence of $\left\{l_{\mathbf{v}^{(n)}}\right\}$ and so of the discrete paths $\left\{\mathbf{v}^{(n)}\right\}$, there is a further subsequence of the linear paths which converges uniformly to a limit path $\gamma \in \arg \min F$, and of the discrete paths in the Hausdorff sense to $S_{\gamma}$.

If $F$ has a unique (up to reparametrization) minimizer $\gamma$, the whole sequence of discrete paths converges, $\lim _{n \rightarrow \infty} d_{\text {haus }}\left(\mathbf{v}^{(n)}, S_{\gamma}\right)=0$. 
2.3. Remarks. We make several comments about the assumptions and related issues.

1. Domain. The requirements that $D$ should be closed and connected are needed for the quasinormal path results in [9] and [16] to hold. Also, a bound on the domain allows the Arzelà-Ascoli equicontinuity criterion to be applied in the compactness property, Lemma 3.7. In addition, the proof of Proposition A.1, on the maximum distance to a nearest neighbor vertex, requires that the domain boundary should be Lipschitz, true for convex domains. The convexity of the domain also ensures that all the linearly interpolated paths are within the domain, and allows comparison with quasinormal ones, which by definition are constrained in the domain. In the $p>1$ case, convexity principally enters in the proof of the limsup inequality, Lemma 3.5, and its requisite propositions. Here, the assumption could perhaps be relaxed in favor of a more complicated construction of the recovery sequence. However, in the $p=1$ case, convexity plays an important role in our compactness arguments (cf. Proposition 3.6 and Section 4.2.1, which rely on the existence of straight-line paths between nearby points in order to bound the number of vertices in an optimal path), and cannot be immediately removed without some a priori control over the distance from discrete optimal paths to the boundary of the domain.

2. Ellipticity of $\rho$. The bound on $\rho$ is useful to compare $v$ to the uniform distribution in the nearest-neighbor map result, Proposition A.1, as well as in bounding the number of points in certain sets in Lemma 4.5. We note, as our approximating costs, $L_{n}, G_{n}, H_{n}$, do not involve density estimators, our results do not depend on the specifics of $\rho$, unlike for "density based distances" discussed in [28].

3. Decay of $\varepsilon_{n}$ (2.6). Intuitively, the rate $\varepsilon_{n}$ cannot vanish too quickly, as then the graph may be disconnected with respect to a positive set of realizations $\left\{X_{i}\right\}$. However, the estimate in (2.6) ensures that the graph $\mathcal{G}_{n}(a, b)$ is connected for all large $n$ almost surely; see Proposition A.1. This is a version of the $\delta$-sampling condition in [7], and is related to connectivity estimates in continuum percolation (cf. [26]). Moreover, we note, the prescribed rate yields in fact that any vertex $X_{i}$ will have degree tending to infinity as $n$ grows, as long as $\rho$ is elliptic: One calculates that the mean number of points in the $\varepsilon_{n}$ ball around $X_{i}$ is of order $n \varepsilon_{n}^{d}$ which grows faster than $\log (n)$.

4. Assumptions (A0)-(A3) on $f$. These are somewhat standard assumptions to treat parametric variational integrals such as $F$ (cf. [9] and [16]), which include the basic case $f(x, v)=|v|^{p}$.

5. Assumption on $p$. The assumption $p \geq 1$ is useful to show existence of quasinormal paths in Proposition A.2, and compactness of minimizers. The case $p<1$ is more problematic in this sense and not discussed here.

6. Extra assumptions in Theorems 2.5 and 2.6. The main difficulty is in showing compactness of optimal $H_{n}$ and $L_{n}$ paths when $p=1$. With respect to Theorem 2.4, when $p>1$, the form of the cost allows a Hölder's inequality argument to deduce equicontinuity of the paths, from which compactness follows using Arzelà-Ascoli's theorem. However, there is no such coercivity when $p=1$. 
Yet, with the additional assumptions, one can approximate a geodesic locally by straight lines. Several geometric estimates on the number of points in small windows around these straight lines are needed to ensure accuracy of the approximation, for which the upper bound on $\varepsilon_{n}$ in (2.12) is useful. In the absence of these assumptions, it is not clear whether one may control the oscillations of the approximating paths.

On the other hand, compactness with respect to the Hausdorff topology, viewing the geodesic as a set of points, might be explored, under less or a different set of assumptions. Perhaps somewhat related, we mention [12], and references therein, which considers Gromov-Hausdorff convergence of the space of probability measures on a random geometric graph in a torus endowed with a discrete Wasserstein metric to the space of probability measures on the torus endowed with the Wasserstein metric.

7. Unique minimizers of $F$. Given that our results achieve their strongest form when $\arg \min F$ consists of a unique minimizing path, perhaps up to reparametrization, we comment on this possibility. Under suitable smoothness conditions on the integrand $f$, uniqueness criteria for ordinary differential equations allow to deduce from the Euler-Lagrange equations for $F$ that there is a unique geodesic between points $a, b$ sufficiently close together (cf. Proposition 5.25 in [9] and Chapter 5 in [8]). On the other hand, for general $a, b$, nonuniqueness may hold depending on the structure of $f$. For instance, one may construct an $f$, satisfying the standing assumptions, with several $F$-minimizing paths, by penalizing portions of $D$ so as to induce forks.

8. $k$-nearest neighbor graphs. It is not clear if our approximation results, say Theorem 2.5, hold with respect to the $k$-nearest-neighbor graph with $k$ boundedthat is the graph formed by attaching edges from a vertex to the nearest $k$ points. For instance, when $\left\{X_{i}\right\}$ is arranged along a fine regular grid, $f(x, v) \equiv|v|, d=2$, and $k=4$, the optimal $H_{n}$ route of moving from the origin to $(1,1)$ is on a staircase path with length $\sim 2$, no matter how refined the grid is, yet the Euclidean distance is $\sqrt{2}$. In this respect, the random geometric graph setting of Theorem 2.5 allows enough choices among nearby points, as long as $\rho$ is elliptic, for the optimal path to approximate the straight line from $(0,0)$ to $(1,1)$. It would be of interest to investigate the extent to which our results extend to $k$-nearest neighbor graphs.

9. Computing optimal paths. In the $p=1$ case, minimization of the functionals $L_{n}, G_{n}$ and $H_{n}$ may be naturally posed as a problem of finding shortest paths in a weighted graph [undirected with symmetric weights, in the case of $L_{n}$ and $G_{n}$, and directed with asymmetric weight $f(x, y-x)$ on the edge from $x$ to $y$ for $H_{n}$ ]. Under such a formulation, finding optimal paths is computationally tractable, even when the number of vertices $n$ is large, for example, by means of Dijkstra's algorithm, which requires $O(m \log n)$ operations, where $m$ is the number of weighted edges in the graph, or via the Euclidean heuristic algorithm of [29] which, when the continuum metric is available to prune the search space, requires $O(n)$ operations. We mention, under the assumption (2.6), $m$ grows faster than $n \log (n)$. 
10. More general kernels and domains. From an applications view, say with respect to the ISOMAP procedure, one presumes that the data lies in a submanifold with a definite, but unknown structure. To estimate distances in the submanifold, one would like to know when approximations via a random sample $\mathcal{X}_{n}$ are consistent, in the most general situation. Also, from a more theoretical perspective, it is natural to ask about the scope of the consistency of the approximations, both of the distances and the geodesic trajectories.

Although, the class of kernels $f$ that we consider generalize those of [7], where $f(x, v)=|v|$, and [28], where $f(x, v)=h(\rho(x))|v|$ and $h$ satisfies several conditions, in applications where the density $\rho$ is not known in advance it would be useful to formulate density dependent versions, as done in [28].

Also, it would be of interest to consider consistency on a more general domain, such as a compact manifold. In this context, the domain $D$ here represents a single patch of a manifold, and the results here on $D$ may be seen as a basis for further advances.

2.4. An example class of kernels. We now describe a class of kernels $f$ satisfying all the assumptions in the article. The following lemma is a case of the Hamel's criterion discussed previously.

LEMMA 2.7. Given the standing assumptions, suppose also, for fixed $x \in D$, that $v \mapsto f(x, v)$ is $C^{2}$ on $\mathbb{R}^{d} \backslash\{0\}$ with positive definite Hessian. Then (Hilb) is satisfied.

Proof. Fix an $x_{0} \in D$. There is a quasinormal minimizer $\gamma \in C^{2}$ where both $c=f\left(x_{0}, \dot{\gamma}(t)\right)=\inf _{\gamma \in \Omega(a, b)} \int_{0}^{1} f\left(x_{0}, \dot{\gamma}(t)\right) d t$ and

$$
c^{2}=f^{2}\left(x_{0}, \dot{\gamma}(t)\right)=\inf _{\gamma \in \Omega(a, b)} \int_{0}^{1} f^{2}\left(x_{0}, \dot{\gamma}(t)\right) d t
$$

for a.e. $0 \leq t \leq 1$ (cf. Proposition 5.25 in [9]). Let $g(v)=f^{2}\left(x_{0}, v\right)$. Then $\gamma$ satisfies the Euler-Lagrange equation $\frac{d}{d t} \nabla_{v} g(\dot{\gamma}(t))=\nabla_{x} g(\dot{\gamma}(t))=0$. In other words, $H \ddot{\gamma}(t)=0$, where $H$ denotes the Hessian of $g$. By assumption, $H$ is positive definite. Hence, $\ddot{\gamma}(t) \equiv 0$, and so $\gamma$ is a parametrization of a straight line.

Recall $\langle\cdot, \cdot\rangle$ denotes the Euclidean inner product on $\mathbb{R}^{d}$.

LEMMA 2.8. Let $x \mapsto M=M(x)$ be a $C^{1}$, strictly elliptic matrix-valued function on D. The kernel $f(x, v)=\langle v, M(x) v\rangle^{1 / 2}$ satisfies the standing assumptions, and also (Lip), (Hilb), (TrIneq) and (Pythag) for all $\alpha>1$.

PROOF. The kernel clearly satisfies the standing assumptions and (Lip). Next, for fixed $x$, the map $v \mapsto f(x, v)$ satisfies the conditions of Lemma 2.7, and so satisfies (Hilb). Also, $v \mapsto f(x, v)$ trivially satisfies (TrIneq). 


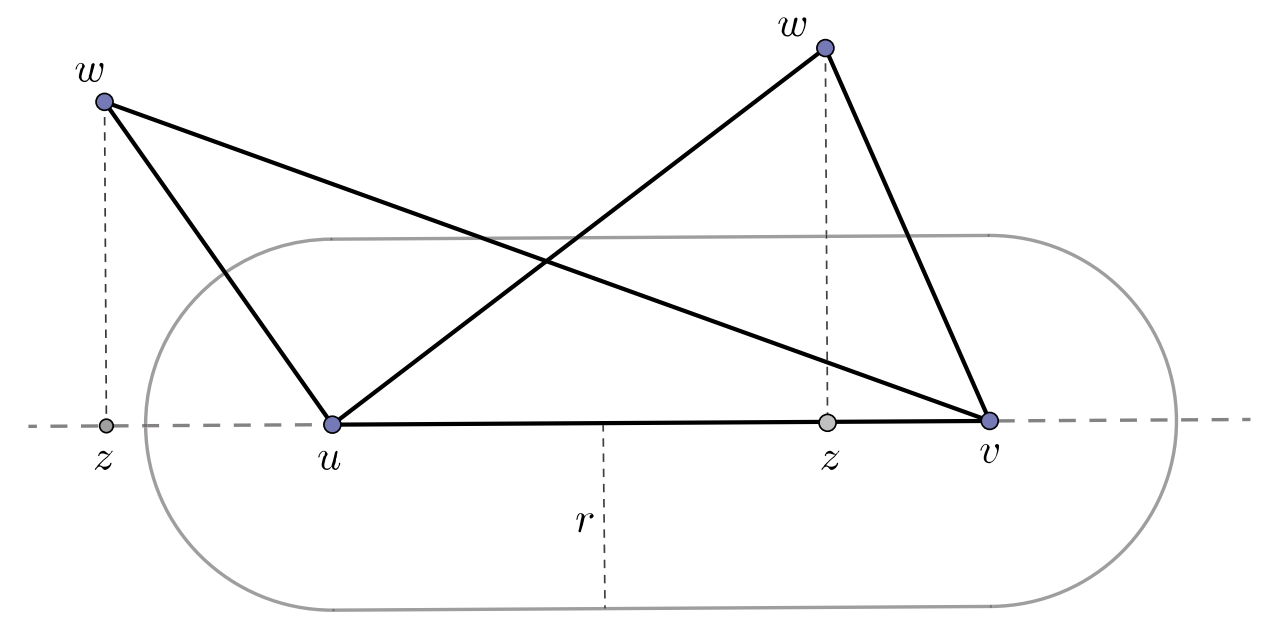

FIG. 3. Geometric argument used in proof of Lemma 2.8 with respect to (Pythag).

We show (Pythag) in the case $f(x, v)=h(x)|v|$, that is $M(x)=h^{2}(x) \operatorname{Id}$, as the notation is easier and all the ideas carry over to the more general case. Consider a right triangle joining $u, w, z$ where $z$ is on the line through $u, v$ (cf. Figure 3).

If $z$ is not on the line segment connecting $u$ and $v$, then either (a) $|u w| \geq$ $|u v|$ and $|w v| \geq r \geq r^{\alpha}$ or (b) $|w v| \geq|u v|$ and $|u w| \geq r \geq r^{\alpha}$. In either case, $h(x)[|u w|+|w v|] \geq h(x)|u v|+m_{1} r^{\alpha}$ and (Pythag) is satisfied.

Suppose now $z$ is on the line segment connecting $u$ and $v$. In the triangle, $u w$ is the hypotenuse, and $w z$ and $u z$ are the legs, such that $|u w|^{2}=|w z|^{2}+|u z|^{2}$. Hence, as $|u w|<1$, all the lengths are less than 1 . We are given that $|w z| \geq r$ and $|u z| \leq|u v| \leq c r^{1 / \alpha}$. Then, as $r \leq|w z|<1$ and $2<\min \{2 \alpha, \alpha+1 / \alpha\}$, we have

$$
\begin{aligned}
|u w|^{2} & =|u z|^{2}+|w z|^{2} \\
& \geq|u z|^{2}+(1 / 9) r^{2 \alpha}+2(1 / 3) r^{\alpha+1 / \alpha} \\
& \geq|u z|^{2}+\left(9 \max \{c, 1\}^{2}\right)^{-1} r^{2 \alpha}+2(3 \max \{c, 1\})^{-1}|u z| r^{\alpha} \\
& =\left(|u z|+(3 \max \{c, 1\})^{-1} r^{\alpha}\right)^{2},
\end{aligned}
$$

and so $|u w| \geq|u z|+(3 \max \{c, 1\})^{-1} r^{\alpha}$.

A similar inequality, $|w v| \geq|v z|+(3 \max \{c, 1\})^{-1} r^{\alpha}$, holds with the same argument. Hence, $h(x)|u w|+h(x)|w v| \geq h(x)|u v|+C\left(m_{1}, c\right) r^{\alpha}$.

3. Proofs of Theorems 2.1, 2.2 and Corollary 2.3. As mentioned in the Introduction, the proof of Theorems 2.1 and 2.2 relies on a Gamma convergence argument. After establishing some basic notation and results on quasinormal minimizers, we present three main proof elements, liminf inequality, limsup inequality and compactness, in the following subsections. Proofs of Theorems 2.1 and 2.2, and Corollary 2.3 are the end of the section. 
3.1. Preliminaries. Define a nearest-neighbor map $T_{n}: D \rightarrow \mathcal{X}_{n}$ where, for $x \in D, T_{n}(x)$ is the point of $\mathcal{X}_{n}$ closest to $x$ with respect to the Euclidean distance. In the event of a tie, we adopt the convention that $T_{n}(x)$ is that nearest neighbor in $\mathcal{X}_{n}$ with the smallest subscript. Since $\mathcal{X}_{n}$ is random, we note $T_{n}$ and the distortion

$$
\left\|T_{n}-\mathrm{Id}\right\|_{\infty}=\sup _{x \in D}\left|T_{n}(x)-x\right|=\left\|T_{n}-\mathrm{Id}\right\|_{\infty}=\sup _{y \in D} \min _{1 \leq i \leq n}\left|X_{i}-y\right|
$$

are also random. In Proposition A.1 of the Appendix, we show for $a, b \in D$ that, almost surely,

the graph $\mathcal{G}_{n}(a, b)$ is connected for all $a, b \in D$ and all large $n$.

Moreover, it is shown that exists a constant $C$ such that almost surely,

$$
\limsup _{n \rightarrow \infty} \frac{\left\|T_{n}-\mathrm{Id}\right\|_{\infty} n^{1 / d}}{(\log n)^{1 / d}} \leq C .
$$

Throughout, we will be working with realizations where both (3.1) and (3.2) are satisfied. Let

$$
A_{\mathrm{NN}} \text { be the probability } 1 \text { event that (3.1) and (3.2) hold. }
$$

We observe, when the decay rate (2.6) on $\varepsilon_{n}$ holds, on the set of realizations $A_{\mathrm{NN}}$, "NN" signifying nearest-neighbor, we have $\lim _{n \rightarrow \infty}\left\|T_{n}-\operatorname{Id}\right\|_{\infty} / \varepsilon_{n}=0$.

To rule out certain degenerate configurations of points, in $d \geq 2$, let

$$
A_{Q} \text { be the event that } X_{k} \notin S_{\gamma_{X_{i}, X_{j}}} \forall \text { distinct } i, j, k \in \mathbb{N} \text {. }
$$

Since the $\left\{X_{i}\right\}$ come from a continuous distribution, and the image of the Lipschitz path $\gamma_{X_{i}, X_{j}}$ in $D \subset \mathbb{R}^{d}$, when $d \geq 2$, is of lower dimension, $A_{Q}$ has probability 1 ; here, "Q" signifies not on quasinormal.

Recall the definitions of quasinormal and linear paths $\gamma_{u, v}$ and $l_{u, v}$.

PROPOSITION 3.1. Let $m_{1}$ and $m_{2}$ be the constants in (2.4). Then for $u, v \in$ $D$,

$$
m_{1}|u-v|^{p} \leq d_{f}(u, v) \leq m_{2}|u-v|^{p} .
$$

Further, the path $\gamma_{u, v}$ satisfies, for $0 \leq s, t \leq 1$, that

$$
\left|\gamma_{u, v}(s)-\gamma_{u, v}(t)\right| \leq\left(m_{2} / m_{1}\right)^{1 / p}|u-v||s-t|
$$

and

$$
\sup _{0 \leq t \leq 1}\left|\gamma_{u, v}(t)-l_{u, v}(t)\right| \leq\left(\left(m_{2} / m_{1}\right)^{1 / p}+1\right)|u-v|
$$


PROOF. For a Lipschitz path $\gamma$ between $u$ and $v$, we have $m_{1}|\dot{\gamma}(t)|^{p} \leq$ $f(\gamma(t), \dot{\gamma}(t)) \leq m_{2}|\dot{\gamma}(t)|^{p}$ by (2.4). Also, $\inf _{\gamma \in \Omega(a, b)} \int_{0}^{1}|\dot{\gamma}(t)|^{p} d t=|b-a|^{p}$ by a standard calculus of variations argument (see also Proposition A.2). So, by taking infimum over $\gamma$, we obtain (3.3).

Suppose now $\gamma=\gamma_{u, v}$ is quasinormal, so that $f(\gamma(t), \dot{\gamma}(t))=c$ for some constant $c$ and a.e. $t$. Integrating, and noting (3.3), gives $c=\int_{0}^{1} f(\gamma, \dot{\gamma}) d t=$ $d_{f}(u, v) \leq m_{2}|u-v|^{p}$. Also, by (2.4), $m_{1}|\dot{\gamma}(t)|^{p} \leq f(\gamma(t), \dot{\gamma}(t))=c$. Hence, $|\dot{\gamma}(t)| \leq\left(m_{2} / m_{1}\right)^{1 / p}|u-v|$ and (3.4) follows.

Finally, to establish (3.5), suppose that there is a $t$ such that $\left|\gamma_{u, v}(t)-l_{u, v}(t)\right|>$ $\left(\left(m_{2} / m_{1}\right)^{1 / p}+1\right)|u-v|$. Then, considering that $\left|l_{u, v}(t)-v\right| \leq|u-v|$, an application of the triangle inequality gives $\left|\gamma_{u, v}(t)-v\right|>\left(m_{2} / m_{1}\right)^{1 / p}|u-v|$. However, by (3.4), $\left|\gamma_{u, v}(t)-v\right|=\left|\gamma_{u, v}(t)-\gamma_{u, v}(1)\right| \leq\left(m_{2} / m_{1}\right)^{1 / p}|u-v||t-1| \leq$ $\left(m_{2} / m_{1}\right)^{1 / p}|u-v|$, a contradiction. Thus, inequality (3.5) holds.

3.2. Liminf inequality. A first step in getting some control over the limit cost $F$ in terms of the discrete costs is the following bound.

LEMMA 3.2 (Liminf inequality). Consider $\gamma \in \Omega(a, b)$, and suppose we have a sequence of paths $\gamma_{n} \in \Omega(a, b)$ such that

$$
\lim _{n \rightarrow \infty} \sup _{0 \leq t \leq 1}\left|\gamma_{n}(t)-\gamma(t)\right|=0 \quad \text { and } \sup _{n} \int_{0}^{1}\left|\dot{\gamma}_{n}\right|^{p} d t<\infty .
$$

Then, $F(\gamma) \leq \liminf _{n \rightarrow \infty} F\left(\gamma_{n}\right)$.

PROOF. A sufficient condition for this inequality, a lower semicontinuity property of $F$, to hold is that $f(x, v)$ be jointly continuous and convex in $v$. See Theorem 3.5 (and the subsequent Remark 2) of [9] for more discussion on this matter.

3.3. Limsup inequality. To make effective use of the liminf inequality, we need to identify a sufficiently rich set of sequences for which a reverse inequality holds. To this end, we develop certain approximations of Lipschitz paths by piecewise linear or piecewise quasinormal paths.

The following result gives a method for recovering an element of $V_{n}(a, b)$ from a suitable element of $\Omega(a, b)$.

Proposition 3.3. Suppose, for constants $c, C$, that $\gamma \in \Omega(a, b)$ satisfies

$$
c \leq \frac{|\gamma(s)-\gamma(t)|}{|s-t|} \leq C,
$$

for all $0 \leq s<t \leq 1$. Let $N=N(n)=\left\lceil K / \varepsilon_{n}\right\rceil$, where $K=C+1$ say, and define $\mathbf{v}=\left(v_{0}, \ldots, v_{N}\right)$ with $v_{0}=a, v_{N}=b$, and $v_{i}=T_{n} \gamma(i / N)$ for $0<i<N$.

Then, with respect to realizations $\left\{X_{i}\right\}$ in $A_{\mathrm{NN}}$, we have $\mathbf{v} \in V_{n}(a, b)$ for all sufficiently large $n$. 
PROOF. To show that $\mathbf{v} \in V_{n}(a, b)$, it is sufficient to verify that consecutive vertices $v_{i-1}$ and $v_{i}$ are connected by an edge in $\mathcal{G}_{n}(a, b)$, or in other words

$$
0<\left|v_{i}-v_{i-1}\right|<\varepsilon_{n} \quad \text { for } i=1, \ldots, N .
$$

We first show that $\left|v_{i}-v_{i-1}\right|<\varepsilon_{n}$. Note that $|\gamma(i / N)-\gamma((i-1) / N)| \leq$ $C / N \leq(C / K) \varepsilon_{n}$ and $C / K<1$. For $1<i<N$, we have

$$
\begin{aligned}
\left|v_{i}-v_{i-1}\right| & =\left|T_{n} \gamma(i / N)-T_{n} \gamma((i-1) / N)\right| \\
& \leq(C / K) \varepsilon_{n}+2\left\|T_{n}-\mathrm{Id}\right\|_{\infty} .
\end{aligned}
$$

Similarly, for segments incident to an endpoint $a$ or $b$, we have

$$
\max \left(\left|v_{1}-v_{0}\right|,\left|v_{N}-v_{N-1}\right|\right) \leq(C / K) \varepsilon_{n}+\left\|T_{n}-\mathrm{Id}\right\|_{\infty} .
$$

In either case, assumption (2.6) on the decay of $\varepsilon_{n}$ implies that, for realizations $\left\{X_{i}\right\}$ in $A_{\mathrm{NN}}$, we have $\left|v_{i}-v_{i-1}\right|<\varepsilon_{n}$ for all $1 \leq i \leq N$ and sufficiently large $n$.

Now, we show that $0<\left|v_{i}-v_{i-1}\right|$. By the Lipschitz lower bound on $\gamma$, we have

$$
|\gamma(i / N)-\gamma((i-1) / N)| \geq c / N>(c /(K+1)) \varepsilon_{n}
$$

for $1 \leq i \leq N$. By a triangle inequality argument, the distance between $v_{i}$ and $v_{i-1}$ is bounded below by $(c /(K+1)) \varepsilon_{n}-2\left\|T_{n}-\mathrm{Id}\right\|_{\infty}$, which on the set $A_{\mathrm{NN}}$, as $\varepsilon_{n}$ satisfies (2.6) and, therefore, vanishes slower than $\left\|T_{n}-\mathrm{Id}\right\|_{\infty}$, is positive for all large $n$.

We now establish some approximation properties obtained by interpolating paths between points in $\mathbf{v}=\left(v_{0}, \ldots, v_{N}\right)$.

Proposition 3.4. Fix $\gamma \in \Omega(a, b)$ satisfying (3.6), and a realization $\left\{X_{i}\right\}$ in $A_{\mathrm{NN}}$. Let $\gamma_{n}=\gamma_{\mathbf{v}}$ and $l_{n}=l_{\mathbf{v}}$, where $N=N(n)$ and $\mathbf{v}=\left(v_{0}, \ldots, v_{N}\right)$ are defined as in Proposition 3.3. Then we obtain

$$
\lim _{n \rightarrow \infty} \sup _{0 \leq t \leq 1}\left|\gamma_{n}(t)-\gamma(t)\right|=0 \quad \text { and } \quad \lim _{n \rightarrow \infty} \sup _{0 \leq t \leq 1}\left|l_{n}(t)-\gamma(t)\right|=0 .
$$

In addition,

$$
\sup _{n}\left\|l_{n}^{\prime}\right\|_{\infty}<\infty \quad \text { and } \quad l_{n}^{\prime}(t) \rightarrow \gamma^{\prime}(t) \quad \text { for a.e. } t \in[0,1] .
$$

PROOF. We first argue that $\lim _{n \rightarrow \infty} \sup _{0 \leq t \leq 1}\left|l_{n}(t)-\gamma(t)\right|=0$. Let $u_{i}=$ $\gamma(i / N)$, and let $\tilde{l}_{n}=l_{\mathbf{u}^{(n)}} \in \Omega(a, b)$ be the piecewise linear interpolation of $\mathbf{u}^{(n)}=$ $\left(u_{0}, \ldots, u_{N}\right)$. As $\gamma$ is Lipschitz and $\lim _{n \rightarrow \infty} N(n)=\infty$, we have $\lim _{n \rightarrow \infty} \sup _{0 \leq t \leq 1}\left|\tilde{l}_{n}(t)-\gamma(t)\right|=0$, and also $\lim _{n \rightarrow \infty} \tilde{l}_{n}^{\prime}(t)=\gamma^{\prime}(t)$ for a.e. $t \in$ $[0,1]$. By construction, $l_{n}(i / N)=v_{i}=T_{n} \gamma(i / N)$ and $\tilde{l}_{n}(i / n)=u_{i}=\gamma(i / N)$ so that

$$
\max _{0 \leq i \leq N}\left|l_{n}(i / N)-\tilde{l}_{n}(i / N)\right| \leq\left\|\operatorname{Id}-T_{n}\right\|_{\infty} .
$$


Then, as $l_{n}$ and $\tilde{l}_{n}$ are piecewise linear, it follows that $\sup _{0 \leq t \leq 1}\left|l_{n}(t)-\tilde{l}_{n}(t)\right| \leq$ $\left\|\mathrm{Id}-T_{n}\right\|_{\infty}$. As $\lim _{n \rightarrow \infty}\left\|\mathrm{Id}-T_{n}\right\|_{\infty}=0$ on $\bar{A}_{\mathrm{NN}}$, we obtain $\lim _{n \rightarrow \infty} \sup _{0 \leq t \leq 1}\left|l_{n}(t)-\gamma(t)\right|=0$.

For $i / N<t<(i+1) / N$, we have

$$
l_{n}^{\prime}(t)=N\left(v_{i+1}-v_{i}\right) .
$$

As $\left|v_{i+1}-v_{i}\right| \leq \varepsilon_{n}$ (Proposition 3.3), it follows that $\left|l_{n}^{\prime}(t)\right| \leq N \varepsilon_{n} \leq K+\varepsilon_{n}$. Hence, $\sup _{n}\left\|l_{n}^{\prime}\right\|_{\infty}<\infty$.

Likewise, $\tilde{l}_{n}^{\prime}(t)=N\left(u_{i+1}-u_{i}\right)$, and so

$$
\left|l_{n}^{\prime}(t)-\tilde{l}_{n}^{\prime}(t)\right| \leq N\left(\left|v_{i+1}-u_{i+1}\right|+\left|v_{i}-u_{i}\right|\right) \leq 2 N\left\|T_{n}-\operatorname{Id}\right\|_{\infty} .
$$

For realizations in $A_{\mathrm{NN}}$, since $N=\left\lceil K / \varepsilon_{n}\right\rceil$ and $\varepsilon_{n}$ satisfies (2.6) and, therefore, vanishes slower than $\left\|T_{n}-\mathrm{Id}\right\|_{\infty}$, we have $\lim _{n \rightarrow \infty} N\left\|T_{n}-\operatorname{Id}\right\|_{\infty}=0$. Hence, $l_{n}^{\prime}(t) \rightarrow \gamma^{\prime}(t)$ for a.e. $t \in[0,1]$.

Now, considering the bound (3.5), it follows that

$$
\sup _{0 \leq t \leq 1}\left|\gamma_{n}(t)-l_{n}(t)\right| \leq \max \left(C\left|v_{0}-v_{1}\right|, \ldots, C\left|v_{N-1}-v_{N}\right|\right) \leq C \varepsilon_{n},
$$

and hence $\left\|\gamma_{n}-\gamma\right\|_{\infty} \rightarrow 0$.

With the above work in place, we proceed to the main result of this subsection.

LEMMA 3.5 (Limsup inequality). Suppose $\gamma \in \Omega(a, b)$ satisfies inequality (3.6). Then, with respect to realizations $\left\{X_{i}\right\}$ in $A_{\mathrm{NN}}$, we may find a sequence of paths $\left\{\gamma_{n}\right\}$ taken either in form for all large $n$ as (1) $\gamma_{n} \in \Omega_{n}^{l}(a, b)$ or (2) $\gamma_{n} \in$ $\Omega_{n}^{\gamma}(a, b)$ such that $\lim _{n \rightarrow \infty} \sup _{0 \leq t \leq 1}\left|\gamma_{n}(t)-\gamma(t)\right|=0$ and

$$
F(\gamma) \geq \limsup _{n \rightarrow \infty} F\left(\gamma_{n}\right)
$$

We remark that the sequence $\left\{\gamma_{n}\right\}$ in the last lemma is called the recovery sequence since the liminf inequality in Lemma 3.2 and the limsup inequality in Lemma 3.5 together imply the $\operatorname{limit}_{n} \lim _{n} F\left(\gamma_{n}\right)=F(\gamma)$.

ProOF OF LemMA 3.5. Let $N=\left\lceil K / \varepsilon_{n}\right\rceil$, where $K=C+1$ say is a constant greater than $C$ in (3.6). Define $v_{0}=a, v_{N}=b$, and $v_{i}=T_{n} \gamma(i / N)$ for $0<i<N$. Then, by Proposition 3.3, $\mathbf{v}=\mathbf{v}^{(n)}=\left(v_{0}, \ldots, v_{N}\right) \in V_{n}(a, b)$.

We now consider paths in case (1). By Proposition 3.4, the interpolated paths $l_{n}=l_{\mathbf{v}} \in \Omega_{n}^{l}(a, b)$ converge uniformly to $\gamma$. Consider the bound

$$
\left|F\left(l_{n}\right)-F(\gamma)\right| \leq \int_{0}^{1}\left|f\left(l_{n}(t), l_{n}^{\prime}(t)\right)-f\left(\gamma(t), \gamma^{\prime}(t)\right)\right| d t .
$$

By Proposition 3.4, $l_{n}^{\prime}$ converges almost everywhere to $\gamma^{\prime}$, and $\sup _{n}\left\|l_{n}^{\prime}\right\|_{\infty}<\infty$. Hence $\left(l_{n}(t), l_{n}^{\prime}(t)\right) \rightarrow\left(\gamma(t), \gamma^{\prime}(t)\right)$ for almost every $t$. Also, $\|\dot{\gamma}\|_{\infty}<C$ by (3.6). 
Since, by (2.4), $f(x, v) \leq m_{2}|v|^{p}$, an application of the bounded convergence theorem yields $\lim _{n \rightarrow \infty}\left|F\left(l_{n}\right)-F(\gamma)\right|=0$. Here, $\left\{l_{n}\right\}$ is the desired recovery sequence.

We now consider case (2). Let $\gamma_{n}=\gamma_{\mathbf{v}} \in \Omega^{\gamma}(a, b)$. By Proposition 3.4, it follows that $\lim _{n \rightarrow \infty} \sup _{0 \leq t \leq 1}\left|\gamma_{n}(t)-\gamma(t)\right|=0$. To show (3.8) for this sequence, note that $F\left(\gamma_{n}\right) \leq F\left(l_{n}\right)$ as $l_{n}$ is a possibly more expensive path connecting the vertices in $\mathbf{v}$. Then, by case (1), $\lim \sup F\left(\gamma_{n}\right) \leq \lim \sup F\left(l_{n}\right) \leq F(\gamma)$.

3.4. Compactness. In this subsection, we consider circumstances under which a sequence of paths $\left\{\gamma_{n}\right\}$, in the context of Theorems 2.1 and 2.2, has a limit point with respect to uniform convergence. Here, the arguments when $p=1$ differ from those when $p>1$.

In particular, consider paths $\gamma_{n}$ where $\int_{0}^{1} f\left(\gamma_{n}(t), \dot{\gamma}_{n}(t)\right) d t$ is uniformly bounded. One has $m_{1}|v|^{p} \leq f(x, v)$ and it follows that $\left\{\gamma_{n}\right\}$ is bounded in the $W^{1, p}$ Sobolev space. When $p>1$, this is sufficient to derive a suitable compactness result. But, when $p=1$, this is no longer the case.

However, when $p=1$, our general outlook is that it is enough to establish a compactness result for sequences of optimal paths, on which certain eccentric possibilities are ruled out.

We begin by considering such compactness when $p=1$, when the paths lie in $\Omega_{n}^{\gamma}(a, b)$. The setting $p>1$ is discussed afterwards.

Proposition 3.6. Suppose $d \geq 2$ and that $p=1$. Then, with respect to realizations $\left\{X_{i}\right\}$ in $A_{\mathrm{NN}} \cap A_{Q}$, for all large $n$, if $\gamma \in \arg \min G_{n}$ and $0 \leq s, t \leq 1$, we have that

$$
|\gamma(s)-\gamma(t)| \leq\left(4 m_{2} / m_{1}^{2}\right) G_{n}(\gamma)|s-t|
$$

PROOF. The path $\gamma \in \Omega_{n}^{\gamma}(a, b)$ is a piecewise quasinormal path of the form $\gamma=\gamma_{\mathbf{v}}$ where $\mathbf{v}=\left(v_{0}, v_{1}, \ldots, v_{m}\right) \in V_{n}(a, b)$. We now try to relate $m$, the number of segments in the path, to $G_{n}(\gamma)$, the path energy. Recall the formula (2.9).

Let $B_{i}$ denote the (open) Euclidean ball of radius $\varepsilon_{n} / 2$ around $v_{i}$. We claim that $\left|B_{i} \cap\left\{v_{0}, \ldots, v_{m}\right\}\right| \leq 2$. To see this, suppose that there are at least 3 points of $\left\{v_{0}, \ldots, v_{m}\right\}$ in $B_{i}$. Let $v_{j}$ and $v_{l}$ denote the points in $B_{i}$ with the smallest and largest index, respectively. By minimality of $\gamma, v_{j} \neq v_{l}$. Let $v_{k}$ denote a third point in $B_{i}$.

As $v_{k}, v_{l} \in B_{i}$, we have $\left|v_{k}-v_{l}\right|<\varepsilon_{n}$, and so these points are connected in the graph. Applying the triangle inequality for $d_{f}$, valid when $p=1$ [cf. (2.5)], and noting on the event $A_{Q}$ that $v_{k} \notin S_{\gamma_{v_{j}, v_{l}}}$, we have

$$
d_{f}\left(v_{j}, v_{l}\right)<d_{f}\left(v_{j}, v_{k}\right)+d_{f}\left(v_{k}, v_{l}\right) \leq d_{f}\left(v_{j}, v_{j+1}\right)+\cdots+d_{f}\left(v_{l-1}, v_{l}\right) .
$$

Thus, the path $\tilde{\gamma}=\gamma_{\mathbf{w}}$, where $\mathbf{w}=\left(v_{0}, \ldots, v_{j}, v_{l}, \ldots, v_{m}\right)$, satisfies $G_{n}(\tilde{\gamma})<$ $G_{n}(\gamma)$. This contradicts the optimality of $\gamma$, and so $\left|B_{i} \cap\left\{v_{0}, \ldots, v_{m}\right\}\right| \leq 2$. 
We may thus cover the vertices of $\gamma$ with balls $\left\{B_{i}\right\}_{i=1}^{m}$, centered on the vertices $\left\{v_{i}\right\}_{i=1}^{m}$, and each of these balls contains at most two vertices. It follows that there is a subcover by $s \geq m / 2$ balls, $\left\{B_{1}^{\prime}, \ldots, B_{s}^{\prime}\right\}$, no two of them containing a common point in $\mathbf{v}$.

A lower bound for $G_{n}(\gamma)$ is found by considering that part of the $G_{n}$-integral contributed to by the portion of the path $\gamma$ in $B_{i}^{\prime}$. Each such portion, if it does not terminate in $B_{i}^{\prime}$, must visit both the center of $B_{i}^{\prime}$ and the boundary $\partial B_{i}^{\prime}$, and hence has Euclidean length at least $\varepsilon_{n} / 2$. Summing over these portions, we obtain

$$
\frac{m \varepsilon_{n}}{4} \leq \sum_{i=1}^{s} \frac{\varepsilon_{n}}{2} \leq L
$$

where $L=\int_{0}^{1}|\dot{\gamma}(t)| d t$ is the Euclidean arc length of $\gamma$.

By (2.4), it follows that

$$
m_{1} \frac{m \varepsilon_{n}}{4} \leq m_{1} L \leq \int_{0}^{t} f(\gamma(t), \dot{\gamma}(t)) d t=G_{n}(\gamma) .
$$

To get a Lipschitz bound for $\gamma$, recall the bound (3.4). Then $\gamma_{v_{i-1}, v_{i}}(m \cdot-i)$ is Lipschitz with constant $\left(m_{2} / m_{1}\right) m\left|v_{i-1}-v_{i}\right|$. It follows that $\gamma$, being the concatenation of these segments, satisfies

$$
\begin{aligned}
|\gamma(s)-\gamma(t)| & =\left|\sum_{r=0}^{q-1} \gamma\left(w_{r}\right)-\gamma\left(w_{r+1}\right)\right| \\
& \leq\left(m_{2} / m_{1}\right) m \max \left(\left|v_{0}-v_{1}\right|, \ldots,\left|v_{m-1}-v_{m}\right|\right) \sum_{r=0}^{q-1}\left|w_{r}-w_{r+1}\right| \\
& \leq\left(m_{2} / m_{1}\right) m \varepsilon_{n}|s-t|,
\end{aligned}
$$

where $s=w_{0}<\cdots<w_{q}=t,\left\{w_{r}\right\}_{r=1}^{q-1} \subset\{j / m\}_{j=1}^{m-1}$ so that $\left|w_{r}-w_{r+1}\right| \leq 1 / m$ for $0 \leq r \leq q-1$. Then, with (3.9), we obtain

$$
|\gamma(t)-\gamma(s)| \leq\left(m_{2} / m_{1}\right) m \varepsilon_{n}|s-t| \leq\left(4 / m_{1}\right)\left(m_{2} / m_{1}\right) G_{n}(\gamma)|s-t|,
$$

completing the proof.

We now prove our compactness property.

LEMMA 3.7 (Compactness Property). (I). Suppose for all large $n$ that either $\gamma_{n} \in \arg \min L_{n}$ or $\gamma_{n} \in \arg \min G_{n}$. Then, for realizations $\left\{X_{i}\right\}$ in $A_{\mathrm{NN}}$, we have $\sup _{n} F\left(\gamma_{n}\right)<\infty$.

(II). Consider now the following cases:

(i) Suppose paths $\gamma_{n} \in \arg \min G_{n}$ for all large $n$.

(ii) Suppose $p>1$ and $\gamma_{n} \in \Omega(a, b)$ for all large $n$ such that $\sup _{n} F\left(\gamma_{n}\right)<\infty$. 
Then, in case (i) when $p>1$, and in case (ii), with respect to realizations $\left\{X_{i}\right\}$ in $A_{\mathrm{NN}}$, we have $\left\{\gamma_{n}\right\}$ is relatively compact for the topology of uniform convergence. For case (i) when $d \geq 2$ and $p=1$, the same conclusion holds with respect to realizations $\left\{X_{i}\right\}$ in $A_{\mathrm{NN}} \cap A_{Q}$.

PROOF. We first prove the bound $\sup _{n} F\left(\gamma_{n}\right)<\infty$ in part (I). Choose a $\tilde{\gamma} \in$ $\Omega(a, b)$, where (3.6) holds, and $F(\tilde{\gamma})<\infty$. By Lemma 3.5, there is a sequence $\left\{\tilde{\gamma}_{n}\right\}$ of either piecewise linear or quasinormal paths such that $\lim _{\sup } \sin _{n \rightarrow \infty} F\left(\tilde{\gamma}_{n}\right) \leq$ $F(\tilde{\gamma})$. Hence, by minimality of $\left\{\gamma_{n}\right\}$, with respect to paths in either $\Omega_{n}^{l}$ or $\Omega_{n}^{\gamma}$, we have

$$
\sup _{n} F\left(\gamma_{n}\right) \leq \sup _{n} F\left(\tilde{\gamma}_{n}\right)<\infty .
$$

We now argue the claims for cases (i) and (ii). In both cases, as $D$ is bounded, the paths $\gamma_{n}:[0,1] \rightarrow D$ are uniformly bounded. To invoke the Arzelà-Ascoli theorem, we must show that $\left\{\gamma_{n}\right\}$ is an equicontinuous family.

In case (i), when $d \geq 2$ and $p=1$, by Lemma 3.6 on realizations in $A_{\mathrm{NN}} \cap A_{Q}$, we have $\left|\gamma_{n}(s)-\gamma_{n}(t)\right| \leq C G_{n}\left(\gamma_{n}\right)|s-t|$, with $C$ independent of $n$. As $G_{n}\left(\gamma_{n}\right)=$ $F\left(\gamma_{n}\right)$, combining with (3.10), it follows that $\left\{\gamma_{n}\right\}$ is equicontinuous.

If $p>1$, with respect to realizations in $A_{\mathrm{NN}}$, (3.10) implies that, if case (i) holds for the sequence, then case (ii) holds.

Without loss of generality, then we focus our attention now on case (ii). Recall, by (2.4), that $m_{1}|v|^{p} \leq f(x, v)$. Let $q$ be the conjugate of $p$, that is, $1 / p+1 / q=1$. Then, for $0 \leq s<t \leq 1$,

$$
\begin{aligned}
\left|\gamma_{n}(s)-\gamma_{n}(t)\right| & \leq \int_{s}^{t}\left|\gamma_{n}^{\prime}(r)\right| d r \leq|t-s|^{1 / q}\left(\int_{0}^{1}\left|\gamma_{n}^{\prime}(t)\right|^{p} d t\right)^{1 / p} \\
& \leq\left(|t-s|^{1 / q} / m_{1}^{1 / p}\right)\left(\int_{0}^{1} f\left(\gamma_{n}(t), \gamma_{n}^{\prime}(t)\right) d t\right)^{1 / p} \\
& =\left(|t-s|^{1 / q} / m_{1}^{1 / p}\right) F\left(\gamma_{n}\right)^{1 / p} .
\end{aligned}
$$

Combining (3.11) and the assumption in case (ii) that $\sup _{n} F\left(\gamma_{n}\right)<\infty$, we have $\left|\gamma_{n}(s)-\gamma_{n}(t)\right| \leq C|t-s|^{1 / q}$ for a constant $C$ independent of $n$, and hence $\left\{\gamma_{n}\right\}$ is equicontinuous.

3.5. Proofs of Theorems 2.1 and 2.2. With the preceding Gamma convergence ingredients in place, the proofs of Theorem 2.1 and 2.2 are similar, and will be given together.

ProOF. Fix a realization $\left\{X_{i}\right\}$ in the probability 1 set $A_{\mathrm{NN}}$. Let $\left\{\gamma_{n}\right\}$ be a sequence of paths such that, for all large $n$, we have either $\gamma_{n} \in \arg \min L_{n}$ or $\gamma_{n} \in$ $\arg \min G_{n}$. Supposing that $\left\{\gamma_{n}\right\}$ has a subsequential $\operatorname{limit}_{k \rightarrow \infty} \lim _{k} \gamma_{n_{k}}=\gamma$, with respect to the topology of uniform convergence, we now argue that $\gamma \in \arg \min F$. 
By the liminf Lemma 3.2, we have

$$
F(\gamma) \leq \liminf _{k \rightarrow \infty} F\left(\gamma_{n_{k}}\right)
$$

Let $\gamma^{*} \in \arg \min F$ where, by inequality (A.4) of Proposition A.2, there exist constants $c_{1}, c_{2}$ such that $c_{1}|s-t| \leq\left|\gamma^{*}(s)-\gamma^{*}(t)\right| \leq c_{2}|s-t|$ for $0 \leq s, t \leq 1$. Hence, by the limsup Lemma 3.5, there exists a sequence $\left\{\gamma_{n}^{*}\right\}$, of either piecewise linear or quasinormal paths, converging uniformly to $\gamma^{*}$ and $\lim _{\sup } \rightarrow \infty F\left(\gamma_{n}^{*}\right) \leq$ $F\left(\gamma^{*}\right)$. Recall that $F(\gamma)=L_{n}(\gamma)$ or $F(\gamma)=G_{n}(\gamma)$ when $\gamma$ is piecewise linear or quasinormal, respectively. Combining with (3.12) and minimality of $\gamma_{n_{k}}$, we have

$$
F(\gamma) \leq \liminf _{k \rightarrow \infty} F\left(\gamma_{n_{k}}\right) \leq \limsup _{k \rightarrow \infty} F\left(\gamma_{n_{k}}^{*}\right) \leq F\left(\gamma^{*}\right)=\min F,
$$

and so $\gamma \in \arg \min F$.

In the case the paths $\left\{\gamma_{n}\right\}$ are piecewise linear, since $F\left(\gamma_{n}\right)=\min L_{n}$, it follows from (3.13) that

$$
\lim _{k \rightarrow \infty} \min L_{n_{k}}=\min F \text {. }
$$

Similarly, when $\left\{\gamma_{n}\right\}$ are piecewise quasinormal, $\lim _{k \rightarrow \infty} \min G_{n_{k}}=\min F$.

Therefore, we have shown that, if a subsequential limit of $\left\{\gamma_{n}\right\}$ exists, it is an optimal continuum path $\gamma \in \arg \min F$.

Consider now Theorem 2.1, where $p>1$ and $\gamma_{n} \in \arg \min L_{n}$, and part (1) of Theorem 2.2 where $p>1$ and $\gamma_{n} \in \arg \min G_{n}$. By the compactness Lemma 3.7, $\sup _{n} F\left(\gamma_{n}\right)<\infty$ and a subsequential limit exists.

Consider now part (2) of Theorem 2.2 where $d \geq 2, p=1$ and $\gamma_{n} \in \arg \min G_{n}$. Suppose that the realization $\left\{X_{i}\right\}$ belongs also to the probability 1 set $A_{Q}$. Then subsequential limits follow again from the compactness Lemma 3.7.

Now, consider any subsequence $\left\{n_{k}\right\}$ of $\mathbb{N}$. Then, by the work above, applied to the sequence $\left\{n_{k}\right\}$, there is a further subsequence $\left\{n_{k_{j}}\right\}$, and a $\gamma \in$ $\arg \min F$, with $\gamma_{n_{k_{j}}} \rightarrow \gamma$ uniformly, in the settings of Theorems 2.1 and 2.2. Moreover, $\lim _{j \rightarrow \infty} \min L_{n_{k_{j}}}=\min F$ when the paths $\left\{\gamma_{n_{k_{j}}}\right\}$ are piecewise linear, and $\lim _{j \rightarrow \infty} \min G_{n_{k_{j}}}=\min F$ when the paths are piecewise quasinormal.

Since this argument is valid for any subsequence $\left\{n_{k}\right\}$ of $\mathbb{N}$, we recover that $\min L_{n} \rightarrow \min F$ or $\min G_{n} \rightarrow \min F$ when respectively the paths are piecewise linear or quasinormal. Finally, if $F$ has a unique minimizer $\gamma$, by considering subsequences again, the whole sequence $\left\{\gamma_{n}\right\}$ must converges uniformly to $\gamma$.

3.6. Proof of Corollary 2.3. Corollary 2.3 is a statement about Hausdorff convergence. In order to adapt the results of Theorems 2.1 and 2.2 to this end, we make the following observation.

PROPOSITION 3.8. Fix a realization $\left\{X_{i}\right\}$ in $A_{\mathrm{NN}}$, and consider a sequence of paths $\left\{\gamma_{n}\right\}$ such that $\gamma_{n}$ for all large $n$ is either in the form (1) $\gamma_{n}=\gamma_{\mathbf{v}^{(n)}}$ or (2) 
$\gamma_{n}=l_{\mathbf{v}^{(n)}}$, where $\mathbf{v}^{(n)} \in V_{n}(a, b)$. Suppose that $\gamma_{n}$ converges uniformly to a limit $\gamma \in \Omega(a, b)$. Then

$$
\lim _{n \rightarrow \infty} d_{\text {haus }}\left(\mathbf{v}^{(n)}, S_{\gamma}\right)=0
$$

PROOF. Write $\mathbf{v}^{(n)}=\left(v_{0}^{(n)}, \ldots, v_{m(n)}^{(n)}\right)$. Since $\gamma_{n} \rightarrow \gamma$ uniformly and $v_{i}^{(n)}=$ $\gamma_{n}\left(\frac{i}{m(n)}\right)$, it follows that

$$
\lim _{n \rightarrow \infty} \max _{1 \leq i \leq m(n)} \inf _{x \in S_{\gamma}}\left|v_{i}^{(n)}-x\right|=0 .
$$

On the other hand, consider $t$ with $i / m(n) \leq t<(i+1) / m(n)$. In case (1),

$$
\left|\gamma(t)-v_{i}^{(n)}\right| \leq|\gamma(t)-\gamma(i / m(n))|+\left\|\gamma_{n}-\gamma\right\|_{\infty} \leq C / m(n)+\left\|\gamma_{n}-\gamma\right\|_{\infty},
$$

where $C$ is the Lipschitz constant of $\gamma$. In case (2), using linearity of the path, $\left|\gamma(t)-v_{i}^{(n)}\right| \leq\left|v_{i+1}^{(n)}-v_{i}^{(n)}\right|+\left\|\gamma_{n}-\gamma\right\|_{\infty} \leq \varepsilon_{n}+\left\|\gamma_{n}-\gamma\right\|_{\infty}$.

Since $\mathbf{v}^{(n)}$ is a path in $V_{n}(a, b)$, one may bound $\varepsilon_{n} m(n) \geq \sum_{i=0}^{m(n)}\left|v_{i}^{(n)}-v_{i+1}^{(n)}\right| \geq$ $|a-b|$, and so $m(n) \geq|a-b| / \varepsilon_{n}$ diverges. Hence, in both cases,

$$
\lim _{n \rightarrow \infty} \sup _{x \in S_{\gamma}} \min _{1 \leq i \leq m(n)}\left|x-v_{i}^{(n)}\right|=0 .
$$

Combining (3.14) and (3.15), it follows that $\lim _{n \rightarrow \infty} d_{\text {haus }}\left(\mathbf{v}^{(n)}, S_{\gamma}\right)=0$.

We now proceed to prove Corollary 2.3.

ProOF OF COROLlary 2.3. We give the argument for the case of piecewise linear optimizers, as the the argument is exactly the same for piecewise quasinormal paths, using Theorem 2.2 instead of Theorem 2.1 below.

Suppose $l_{n}=l_{\mathbf{v}^{(n)}} \in \arg \min L_{n}$ is a sequence of paths where $\mathbf{v}^{(n)} \in V_{n}(a, b)$. By Theorem 2.1, with respect to a probability 1 set of realizations $\left\{X_{i}\right\}$, any subsequence of $\left\{l_{n}\right\}$ has a further subsequence $\left\{l_{n_{k}}\right\}$ which converges uniformly to a $\gamma \in \arg \min F$. By Proposition 3.8, it follows that $\lim _{k \rightarrow \infty} d_{\text {haus }}\left(\mathbf{v}^{\left(n_{k}\right)}, S_{\gamma}\right)=0$.

Suppose now that $F$ has a unique (up to reparametrization) minimizer $\gamma$. Note that $S_{\gamma}$ is invariant under reparametrization of $\gamma$. Then we conclude that all limit points of $\left\{v^{(n)}\right\}$ correspond to $S_{\gamma}$, and hence the whole sequence $v^{(n)}$ converges to $S_{\gamma}, \lim _{n \rightarrow \infty} d_{\text {haus }}\left(v^{(n)}, S_{\gamma}\right)=0$.

4. Proofs of Theorems 2.4, 2.5 and 2.6. The proofs of Theorems 2.4, 2.5 and 2.6 all make use of Theorems 2.1 and 2.2 in comparing the costs $H_{n}$ and $L_{n}$ to $G_{n}$. When $p=1$, as with respect to Theorem 2.2 , the arguments in Theorems 2.5 and 2.6 are more involved, especially with respect to the minimal cost $H_{n}$-path convergence, where several geometric estimates are used to show a compactness principle.

We begin with the following useful fact. 
Proposition 4.1. Suppose $U, W, C: X \rightarrow \mathbb{R}$ are functions such that $|U(x)-W(x)| \leq C(x)$ for all $x \in X$. If $U\left(x_{U}\right)=\min U$ and $W\left(x_{W}\right)=\min W$ then

$$
-C\left(x_{U}\right) \leq \min U-\min W \leq C\left(x_{W}\right) .
$$

Proof. For any $y \in X$, we have $\min U=U\left(x_{U}\right) \leq U(y) \leq W(y)+C(y)$. Taking $y=x_{W}$ gives $\min U \leq \min W+C\left(x_{W}\right)$. The other inequality follows similarly.

4.1. Proof of Theorem 2.4. Suppose $\mathbf{v}=\left(v_{0}, v_{1}, \ldots, v_{m}\right) \in V_{n}(a, b)$. Then, for $0 \leq i \leq m-1$, (Lip) and the observation that neighbors $v_{i}, v_{i+1}$ in the $\varepsilon_{n}$-graph satisfy $\left|v_{i+1}-v_{i}\right| \leq \varepsilon_{n}$, implies

$$
\begin{aligned}
& \mid \int_{\frac{i}{m}}^{\frac{i+1}{m}} f\left(v_{i}+m\left(s-\frac{i}{m}\right)\left(v_{i+1}-v_{i}\right), m\left(v_{i+1}-v_{i}\right)\right) d s \\
& \quad-\frac{1}{m} f\left(v_{i}, m\left(v_{i+1}-v_{i}\right)\right) \mid \\
& \quad \leq \frac{c}{m}\left|v_{i+1}-v_{i}\right|\left(m\left|v_{i+1}-v_{i}\right|\right)^{p} \leq \frac{c \varepsilon_{n}}{m}\left|v_{i+1}-v_{i}\right|^{p} m^{p} .
\end{aligned}
$$

Recall formulas (2.8) and (2.11). Summing over $i$ gives the following estimate relating $L_{n}$ and $H_{n}$ :

$$
\left|L_{n}\left(l_{\mathbf{v}}\right)-H_{n}(\mathbf{v})\right| \leq \frac{c \varepsilon_{n}}{m} \sum_{i=0}^{m-1}\left|v_{i+1}-v_{i}\right|^{p} m^{p} .
$$

Applying (2.4), the right-hand side of (4.1) can be bounded above in terms of both $L_{n}\left(l_{\mathbf{v}}\right)$ and $H_{n}(\mathbf{v})$. Hence, with $c^{\prime}=c m_{1}^{-1}$,

$$
\left|L_{n}\left(l_{\mathbf{v}}\right)-H_{n}(\mathbf{v})\right| \leq c^{\prime} \varepsilon_{n} \min \left(L_{n}\left(l_{\mathbf{v}}\right), H_{n}(\mathbf{v})\right) .
$$

Suppose $l_{\mathbf{v}^{(n)}} \in \arg \min L_{n}$ and $\mathbf{w}^{(n)} \in \arg \min H_{n}$. An immediate consequence of (4.2) and Proposition 4.1 is

$$
\begin{aligned}
-c^{\prime} \varepsilon_{n} \min L_{n} & \leq-c^{\prime} \varepsilon_{n} \min \left(L_{n}\left(l_{\left.\mathbf{v}^{(n)}\right)}, H_{n}\left(\mathbf{v}^{(n)}\right)\right)\right. \\
& \leq \min L_{n}-\min H_{n} \\
& \leq c^{\prime} \varepsilon_{n} \min \left(L_{n}\left(l_{\mathbf{w}^{(n)}}\right), H_{n}\left(\mathbf{w}^{(n)}\right)\right) \leq c^{\prime} \varepsilon_{n} \min H_{n} .
\end{aligned}
$$

By Theorem 2.1, we have $\lim _{n \rightarrow \infty} \min L_{n}=\min F$ for almost all realizations $\left\{X_{i}\right\}$ (those in $A_{\mathrm{NN}}$ as the proof shows). Then $\min H_{n} \leq\left(1+c^{\prime} \varepsilon_{n}\right) \min L_{n}$ and so $\lim \sup \min H_{n} \leq \min F$ a.s. In particular, as $\min F<\infty$, we have $\sup _{n} \min H_{n}<$ $\infty$ a.s.

On the other hand, $\min H_{n} \geq \min L_{n}-c^{\prime} \varepsilon_{n} \min H_{n}$ a.s. As $\sup _{n} \min H_{n}<\infty$, we observe that $\lim \inf \min H_{n} \geq \min F$ a.s. Hence, $\min H_{n} \rightarrow \min F$ a.s. This finishes one part of Theorem 2.4. 
To address the others, consider $l_{\mathbf{w}^{(n)}}$, the piecewise linear interpolation of $\mathbf{w}^{(n)}$. By (4.2), we have

$$
L_{n}\left(l_{\mathbf{w}^{(n)}}\right) \leq\left(1+c^{\prime} \varepsilon_{n}\right) H_{n}\left(\mathbf{w}^{(n)}\right)=\left(1+c^{\prime} \varepsilon_{n}\right) \min H_{n} .
$$

Moreover, noting the optimality of $l_{\mathbf{v}}(n)$ and $\mathbf{w}^{(n)}$ gives

$$
L_{n}\left(l_{\mathbf{v}^{(n)}}\right) \leq L_{n}\left(l_{\mathbf{w}^{(n)}}\right) \leq\left(c^{\prime} \varepsilon_{n}+1\right) H_{n}\left(\mathbf{v}^{(n)}\right) .
$$

Another application of (4.2) yields

$$
H_{n}\left(\mathbf{v}^{(n)}\right) \leq c^{\prime} \varepsilon_{n} L_{n}\left(l_{\mathbf{v}^{(n)}}\right)+L_{n}\left(l_{\mathbf{v}^{(n)}}\right) .
$$

Hence, the left-hand side of (4.3) is bounded as

$$
\begin{aligned}
\min L_{n} & =L_{n}\left(l_{\mathbf{v}^{(n)}}\right) \leq L_{n}\left(l_{\mathbf{w}^{(n)}}\right) \leq\left(c^{\prime} \varepsilon_{n}+1\right)^{2} L_{n}\left(\mathbf{v}^{(n)}\right) \\
& =\left(c^{\prime} \varepsilon_{n}+1\right)^{2} \min L_{n} .
\end{aligned}
$$

Hence, as $\min L_{n} \rightarrow \min F$ a.s., we have

$$
\min F=\lim _{n \rightarrow \infty} L_{n}\left(l_{\mathbf{v}^{(n)}}\right)=\lim _{n \rightarrow \infty} L_{n}\left(l_{\mathbf{w}^{(n)}}\right) \quad \text { a.s. }
$$

We also observe, as a consequence, that $\sup _{n} L_{n}\left(l_{\mathbf{w}^{(n)}}\right)<\infty$ a.s.

Given that $p>1$, by the compactness Lemma 3.7, with respect to realizations $\left\{X_{i}\right\}$ in the probability 1 set $A_{\mathrm{NN}}$, any subsequence of $\left\{l_{\mathbf{w}^{(n)}}\right\}$ has a further uniformly convergent subsequence to a limit $\tilde{\gamma} \in \Omega(a, b)$. By the liminf Lemma 3.2, $F(\tilde{\gamma}) \leq \liminf _{n \rightarrow \infty} L_{n}\left(l_{\mathbf{w}^{(n)}}\right)$ a.s. Finally, by $(4.5)$, it follows that $F(\tilde{\gamma})=\min F$ and so $\tilde{\gamma} \in \arg \min F$. Consequently, if $F$ has a unique minimizer $\gamma$, then the whole sequence $\left\{l_{\mathbf{w}^{(n)}}\right\}$ converges uniformly almost surely to it.

The proofs of statements about Hausdorff convergence follow the same arguments as given for Corollary 2.3, and are omitted.

4.2. Proof of Theorems 2.5 and 2.6. We prove Theorems 2.5 and 2.6 in two parts.

ProOF. First, we prove in Proposition 4.3 that the minimal costs of $H_{n}$ and $L_{n}$ converge to $\min F$, making use of comparisons with quasinormal paths, for which we have control in Theorem 2.2.

Second, in Proposition 4.9 in Section 4.2.2, we show that the minimizing paths converge in the various senses desired. A main tool in this proof is a compactness property (Proposition 4.6), for minimal $H_{n}$ and $L_{n}$-paths when $p=1$, shown in Section 4.2.1.

To supply the proofs of the desired propositions, we now obtain an useful estimate between the cost of a quasinormal path and a linear one. 
Proposition 4.2. Suppose $d \geq 2, p=1$, and that $f$ also satisfies (Lip) and (Hilb). For $a, b \in D$ such that $|b-a| \leq 1$, there is a constant $c_{1}$ such that

$$
\left|d_{f}(a, b)-f(a, b-a)\right| \leq c_{1}|b-a|^{2} .
$$

In particular, as $d_{f}(a, b)=\int_{0}^{1} f(\gamma(t), \dot{\gamma}(t)) d t$ for the quasinormal path $\gamma=\gamma_{a, b}$ connecting $a$ and $b$, we have

$$
\left|\int_{0}^{1} f(\gamma(t), \dot{\gamma}(t)) d t-f(a, b-a)\right| \leq c_{1}|b-a|^{2} .
$$

ProOF. By (2.4), for a Lipschitz path $\beta$ from $a$ to $b$, we have

$$
m_{1} \int_{0}^{1}|\dot{\beta}(t)| d t \leq \int_{0}^{1} f(\beta(t), \dot{\beta}(t)) d t \leq m_{2} \int_{0}^{1}|\dot{\beta}(t)| d t .
$$

Optimizing over $\beta$, noting that the infima of the left, right integrals is the arclength $|b-a|$ and the infima of the center one is in terms of the quasinormal path, we recover that $m_{1}|b-a| \leq \int_{0}^{1} f(\gamma(t), \dot{\gamma}(t)) d t \leq m_{2}|b-a|$. By (2.4) again, we have that the arc length of $\gamma$ satisfies $\int_{0}^{1}|\dot{\gamma}(t)| d t \leq\left(m_{2} / m_{1}\right)|b-a|$. In particular, the path $\gamma$ is constrained in the Euclidean ball $B$ around $a$ of radius $\left(m_{2} / m_{1}\right)|b-a|$. Note also that the minimizing Euclidean path $\tilde{\gamma}$, with constant speed $|b-a|$ on the straight line from $a$ to $b$ in times $0 \leq t \leq 1$, is also constrained in this ball.

Now, for a Lipschitz path $\beta$, constrained in the ball $B$, expand

$$
\int_{0}^{1} f(\beta(t), \dot{\beta}(t)) d t=\int_{0}^{1} f(a, \dot{\beta}(t)) d t+\int_{0}^{1}(f(\beta(t), \dot{\beta}(t))-f(a, \dot{\beta}(t)) d t .
$$

As the paths are in $B$, by (Lip), with respect to a Lipschitz constant $C$,

$$
\begin{aligned}
|f(\beta(t), \dot{\beta}(t))-f(a, \dot{\beta}(t))| & \leq C|\beta(t)-a||\dot{\beta}(t)| \\
& \leq C\left(m_{2} / m_{1}\right)|b-a||\dot{\beta}(t)| .
\end{aligned}
$$

Therefore, with respect to Lipschitz paths $\beta$ constrained in $B$,

$$
\left|\int_{0}^{1} f(\beta(t), \dot{\beta}(t)) d t-\int_{0}^{1} f(a, \dot{\beta}(t)) d t\right| \leq C\left(m_{2} / m_{1}\right)|b-a| \int_{0}^{1}|\dot{\beta}(t)| d t .
$$

Note by condition (Hilb) that, for the cost with respect to $f(a, \cdot)$, straight lines are geodesics, and in particular $\tilde{\gamma}(t)=(1-t) a+t b$ is optimal. Hence, the minimal $F$-cost, with respect to $f(a, \cdot)$, of moving from $a$ to $b$, given invariance to parametrization when $p=1$, is $f(a, b-a)$.

Then, by Proposition 4.1 applied to the two functionals of $\beta$ on the left-hand sides, we obtain

$$
\begin{aligned}
\left|d_{f}(a, b)-f(a, b-a)\right| & \leq C\left(m_{2} / m_{1}\right)|b-a| \max \left[\int_{0}^{1}|\dot{\gamma}(t)| d t, \int_{0}^{1}|\dot{\tilde{\gamma}}(t)| d t\right], \\
& \leq C\left(m_{2} / m_{1}\right)^{2}|b-a|^{2},
\end{aligned}
$$

noting the arc length bounds of $\gamma=\gamma_{a, b}$ and $\tilde{\gamma}$ above. 
Proposition 4.3. Suppose $d \geq 2, p=1$, and that $f$ also satisfies (Lip) and (Hilb). With respect to realizations $\left\{X_{i}\right\}$ in $A_{\mathrm{NN}} \cap A_{Q}$, the minimum values of $H_{n}$ and $L_{n}$ converge to the minimum of $F$,

$$
\lim _{n \rightarrow \infty} \min _{v \in V_{n}(a, b)} H_{n}(v)=\lim _{n \rightarrow \infty} \min _{\gamma \in \Omega_{n}^{l}(a, b)} L_{n}(\gamma)=\min _{\gamma \in \Omega(a, b)} F(\gamma) .
$$

Proof. Consider the energies $G_{n}$ and $H_{n}$ in (2.9) and (2.11). For $\gamma=\gamma_{\mathbf{v}}$, the piecewise quasinormal path through the vertices $\mathbf{v}=\left(v_{0}, v_{1}, \ldots, v_{m}\right) \in V_{n}(a, b)$, we have, noting $p=1$, that

$$
G_{n}(\gamma)=\sum_{i=0}^{m-1} \int_{0}^{1} f\left(\gamma_{i}(t), \dot{\gamma}_{i}(t)\right) d t
$$

where $\gamma_{i}=\gamma_{v_{i}, v_{i+1}}$ is a quasinormal path from $v_{i}$ to $v_{i+1}$.

An application of Proposition 4.2, noting that $\left|v_{i+1}-v_{i}\right| \leq \varepsilon_{n}$, gives

$$
\left|\int_{0}^{1} f\left(\gamma_{i}(t), \dot{\gamma}_{i}(t)\right) d t-f\left(v_{i}, v_{i+1}-v_{i}\right)\right| \leq c_{1} \varepsilon_{n}\left|v_{i+1}-v_{i}\right| .
$$

Summing this over $i$ gives

$$
\begin{aligned}
\left|G_{n}(\gamma)-H_{n}(\mathbf{v})\right| & \leq c_{1} \varepsilon_{n} \sum_{i=0}^{m-1}\left|v_{i+1}-v_{i}\right| \\
& \leq c_{1} m_{1}^{-1} \varepsilon_{n} \min \left(G_{n}(\gamma), H_{n}(\mathbf{v})\right),
\end{aligned}
$$

where the last inequality follows from applying (2.4) to both $G_{n}$ and $H_{n}$.

Recall the energy $L_{n}$ in (2.8). Similarly, and more directly, using (Lip), we have for a linear path $l=l_{\mathbf{v}} \in \Omega_{n}^{l}(a, b)$ through vertices $\mathbf{v}=\left(v_{0}, \ldots, v_{m}\right) \in V_{n}(a, b)$ that

$$
\left|\int_{0}^{1} f\left(l_{i}(t), i_{i}(t)\right) d t-f\left(v_{i}, v_{i+1}-v_{i}\right)\right| \leq c_{1} \varepsilon_{n}\left|v_{i+1}-v_{i}\right|,
$$

where $l_{i}=l_{v_{i}, v_{i+1}}$ is the linear path from $v_{i}$ to $v_{i+1}$ with slope $v_{i+1}-v_{i}$. Summing over $i$, using (2.4), we obtain

$$
\left|L_{n}(l)-H_{n}(\mathbf{v})\right| \leq c_{1} \varepsilon_{n} \sum_{i=0}^{m-1}\left|v_{i+1}-v_{i}\right| \leq c_{1} m_{1}^{-1} \varepsilon_{n} \min \left(L_{n}(l), H_{n}(\mathbf{v})\right) .
$$

We now reprise some of the argument for Theorem 2.4. A consequence of (4.6) and Proposition 4.1 is

$$
-c_{1} m_{1}^{-1} \varepsilon_{n} \min G_{n} \leq \min G_{n}-\min H_{n} \leq c_{1} m_{1}^{-1} \varepsilon_{n} \min H_{n} .
$$

Hence, $\sup _{n} \min H_{n}<\sup _{n}\left(1+c_{1} m_{1}^{-1} \varepsilon_{n}\right) \min G_{n}$. By Theorem 2.2, as seen in its proof, for realizations in the probability 1 set $A_{\mathrm{NN}} \cap A_{Q}$, we have 
$\lim _{n \rightarrow \infty} \min G_{n}=\min F<\infty$. We conclude that also $\lim _{n \rightarrow \infty} \min H_{n}=\min F$ a.s.

Now, we can repeat this same argument with $L_{n}$ and (4.7) in place of $G_{n}$ and (4.6), using now $\min H_{n} \rightarrow \min F$ a.s., to conclude that also $\min L_{n}$ converges to $\min F$ a.s.

4.2.1. Compactness property. When $d \geq 2$ and $p=1$, analogous to Lemma 3.6, we formulate now a compactness property for minimal paths $w^{(n)} \in$ $\arg \min H_{n}$ and $l_{\mathbf{v}^{(n)}} \in \arg \min L_{n}$.

It will be useful to consider a partition of $D$ by a regular grid. Let $z \in \mathbb{Z}^{d}$ and let $\square_{n, z}$ be the intersection of the box $\prod_{i=1}^{d}\left[z_{i} \tau_{n},\left(z_{i}+1\right) \tau_{n}\right)$ with $D$, where $\tau_{n}=$ $\varepsilon_{n} / \sqrt{d}$. We will refer to these sets as "boxes," with the understanding that the boundary of $D$ results in some of these being irregularly shaped. Regardless, each $\square_{n, z}$ has diameter at most $\varepsilon_{n}$, and so points of $\left\{X_{i}\right\}_{i=1}^{n}$ in $\square_{n, z}$ are all connected in the random geometric graph.

We first state a bound on the number of boxes visited by an optimal path.

LemMA 4.4. Suppose $d \geq 2, p=1$, and that $f$ also satisfies (Lip) and (Hilb). Suppose $\mathbf{w} \in \arg \min H_{n}$ and $l_{\mathbf{v}} \in \arg \min L_{n}$ are optimal paths. Then, for realizations $\left\{X_{i}\right\}$ in $A_{\mathrm{NN}} \cap A_{Q}$, for all large $n$, the number of distinct boxes $\left\{\square_{n, z}\right\}_{z \in \mathbb{Z}^{d}}$ visited by $\mathbf{w}$ and $\mathbf{v}$ is bounded by $C / \varepsilon_{n}$, where $C=C(d, f)$.

We also give a bound on the number of points of an optimal path in a box.

LEMMA 4.5. Consider the assumptions of the second parts of Theorems 2.5 and 2.6. Suppose $\mathbf{w} \in \arg \min H_{n}$ is an optimal path. Then, with respect to realizations $\left\{X_{i}\right\}$ in a probability 1 subset of $A_{\mathrm{NN}}$, for all large $n$, there is a constant $K=K(d, \rho, \alpha)$, such that $\left|\left\{w_{j}\right\}_{j=1}^{n} \cap \square_{n, z}\right| \leq K$ for all $z \in \mathbb{Z}^{d}$.

Suppose now $l_{\mathbf{v}} \in \arg \min L_{n}$. Then the same statement above holds with $\mathbf{v}$ in place of $\mathbf{w}$.

With these ingredients, we consider the desired compactness property.

PROPOSITION 4.6. Consider the assumptions in the second parts of Theorems 2.5 and 2.6. Suppose $\mathbf{w}^{(n)} \in \arg \min H_{n}$, and consider the piecewise linear interpolations $l_{n}=l_{\mathbf{w}^{(n)}}$. Then, with respect to a realizations $\left\{X_{i}\right\}_{i \geq 1}$ in a probability 1 subset of $A_{\mathrm{NN}} \cap A_{Q}$, the sequence $\left\{l_{n}\right\}$ is relatively compact for the topology of uniform convergence.

Suppose now $l_{\mathbf{v}(n)} \in \arg \min L_{n}$. Then, the same conclusion holds for the optimal linear interpolations $\left\{l_{\mathbf{v}^{(n)}}\right\}$.

PROOF. We show that the sequence $\left\{l_{n}\right\}$ is equicontinuous for almost all realizations in $A_{\mathrm{NN}}$. As the paths belong to a bounded set $D$, the proposition would then follow from the Arzelà-Ascoli criterion. 
Consider the partition of $D$ by boxes $\left\{\square_{n, z}\right\}_{z \in \mathbb{Z}^{d}}$. By Lemma 4.4, the number of boxes visited by $\mathbf{w}^{(n)}$ and $\mathbf{v}^{(n)}$ is bounded by $C / \varepsilon_{n}$ a.s., for all large $n$, where $C=C(f, d)$. By Lemma 4.5 , the number of vertices in $\mathbf{w}^{(n)}$ and $\mathbf{v}^{(n)}$ in a box is a.s. bounded by a constant $K=K(d, \rho, \alpha)$ for all large $n$. Thus, the maximum number $k_{n}$ of points in $\mathbf{w}^{(n)}$ and $\mathbf{v}^{(n)}$ is a.s. bounded,

$$
k_{n} \leq K C / \varepsilon_{n} .
$$

Since $\left|w_{i+1}^{(n)}-w_{i}^{(n)}\right|,\left|v_{i+1}^{(n)}-v_{i}^{(n)}\right| \leq \varepsilon_{n}$, we obtain $\sup _{i} k_{n}\left|w_{i+1}^{(n)}-w_{i}^{(n)}\right| \leq K C$ and $\sup _{i} k_{n}\left|v_{i+1}^{(n)}-v_{i}^{(n)}\right| \leq K C$ a.s. for all large $n$.

This implies a.s. that the piecewise linear paths $l_{n}$ and $l_{\mathbf{v}^{(n)}}$ are Lipschitz, with respect to the fixed constant $K C$, for all large $n$, and so in particular equicontinuous. Indeed, for $l_{n}=l_{\mathbf{w}^{(n)}}$, where say $\mathbf{w}^{(n)}=\left(w_{0}^{(n)}, \ldots, w_{k_{n}}^{(n)}\right)$, consider the part of the path connecting $w_{i}^{(n)}$ and $w_{i+1}^{(n)}$ from times $i / k_{n}$ to $(i+1) / k_{n}$, namely $l_{n}(t)=$ $w_{i}^{(n)}\left(i+1-k_{n} t\right)+w_{i+1}^{(n)}\left(k_{n} t-i\right)$. Then we have $\left|\dot{l}_{n}(t)\right|=k_{n}\left|w_{i+1}^{(n)}-w_{i}^{(n)}\right| \leq$ $k_{n} \varepsilon_{n} \leq K C$. The same argument holds for the paths $l_{\mathbf{v}^{(n)}}$.

We now prove the lemmas used in the proof Proposition 4.6.

ProOF OF LEMma 4.4. Any visit of the path $\mathbf{w}$ or $\mathbf{v}$ to $2^{d}+1$ distinct boxes has a Euclidean length of at least $\varepsilon_{n} / \sqrt{d}$, since not all $2^{d}+1$ boxes can be adjacent. Recalling (2.4), and the formulas (2.8) and (2.11), such a visitation therefore has a $H_{n}$ cost or $L_{n}$ cost of at least $m_{1} \varepsilon_{n} / \sqrt{d}$. So, we may bound the number of boxes visited by $\mathbf{w}$ or $\mathbf{v}$ by $C^{\prime} H_{n}(\mathbf{w}) / \varepsilon_{n}$, where $C^{\prime}=\left(2^{d}+1\right) \sqrt{d} / m_{1}$ depends on the dimension and $f$, but not on the path $\mathbf{w}$ or $\mathbf{v}$. Recalling Proposition 4.3 , we have with respect to realizations in $A_{\mathrm{NN}} \cap A_{Q}$ that $\lim \min H_{n}=\lim \min L_{n}=\min F<$ $\infty$. The lemma then follows with say $C=2 C^{\prime} \min F$.

Before coming to the proof of Lemma 4.5, we need some estimates. The next result shows that optimal paths $\mathbf{w} \in \arg \min H_{n}$ and $l_{\mathbf{v}} \in \arg \min L_{n}$ cannot have "long necks," and gives a bound on the number of points nearby an edge in the graph.

LEMMA 4.7. Suppose $d \geq 2$ and $p=1$. Fix a realization $\left\{X_{i}\right\}$ in $A_{\mathrm{NN}}$. Suppose $\mathbf{w} \in \arg \min H_{n}$ is an optimal path. If $i<j$ is such that $\left|w_{i}-w_{j}\right|<\varepsilon_{n}$, then

$$
w_{k} \in B\left(w_{i}, C \varepsilon_{n}\right) \quad \text { for } i \leq k \leq j
$$

where $C=2\left(m_{2} / m_{1}\right)$.

Further, let $\Theta_{n}=\sup _{i, j:\left|w_{i}-w_{j}\right|<\varepsilon_{n}}|i-j|$, and suppose $\varepsilon_{n}=n^{-\delta}$, where $\delta>$ $1 /(\beta+d)$ and $\beta>0$. Then, with respect to realizations in a probability 1 subset of $A_{\mathrm{NN}}$, for all large $n$, we have

$$
\Theta_{n} \leq \varepsilon_{n}^{-\beta}
$$


Suppose now $l_{\mathbf{v}} \in \arg \min L_{n}$. The same conclusions (4.8) and (4.9) hold with $\mathbf{v}$ in place of $\mathbf{w}$.

PROOF. We first show (4.8). If one of the points $\left\{w_{k}\right\}_{k=i}^{j}$ is more than an Euclidean distance $2\left(m_{2} / m_{1}\right) \varepsilon_{n}$ away from $w_{i}$, we have $\sum_{k=i}^{j-1}\left|w_{k+1}-w_{k}\right| \geq$ $2\left(m_{2} / m_{1}\right) \varepsilon_{n}$. Then, recalling (2.4), we have

$$
\begin{aligned}
\sum_{k=i}^{j-1} f\left(w_{k}, w_{k+1}-w_{k}\right) & \geq m_{1} \sum_{k=i}^{j-1}\left|w_{k+1}-w_{k}\right| \\
& \geq m_{1}\left(2 m_{2} / m_{1}\right) \varepsilon_{n} \\
& \geq 2 m_{2}\left|w_{j}-w_{i}\right| \geq 2 f\left(w_{i}, w_{j}-w_{i}\right) .
\end{aligned}
$$

But, this implies that the path connecting $w_{i}$ and $w_{j}$ in one step would be less costly, with respect to $H_{n}$, than w. Since w was taken to be minimal, all points $\left\{w_{k}\right\}_{k=i}^{j}$ therefore must belong to $B\left(w_{i}, 2\left(m_{2} / m_{1}\right) \varepsilon_{n}\right)$.

Suppose now $l_{\mathbf{v}} \in \arg \min L_{n}$ and recall the form of $L_{n}$ when $p=1$ in (2.8). Similarly, if one of the points $\left\{v_{k}\right\}_{k=i}^{j}$ is away from $v_{i}$ by $2\left(m_{2} / m_{1}\right) \varepsilon_{n}$, we have

$$
\begin{aligned}
& \sum_{k=i}^{j-1} \int_{0}^{1} f\left(l_{v_{k}, v_{k+1}}(t), v_{k+1}-v_{k}\right) d t \\
& \quad \geq m_{1} \sum_{k=i}^{j-1}\left|v_{k+1}-v_{k}\right| \\
& \quad \geq 2 m_{2} \varepsilon_{n} \geq 2 m_{2}\left|v_{j}-v_{i}\right| \geq 2 \int_{0}^{1} f\left(l_{v_{i}, v_{j}}(t), v_{j}-v_{i}\right) d t
\end{aligned}
$$

also a contradiction of minimality of $l_{\mathbf{v}}$.

We now consider (4.9). The proof here is a count bound with respect to $\mathbf{w}$. The argument with respect to $\mathbf{v}$ is exactly the same with $\mathbf{v}$ in place of $\mathbf{w}$.

First, let $N_{i, n}$ be the number of points $\left\{X_{i}\right\}_{i=1}^{n}$ distinct from $X_{i}$ in the ball $B\left(X_{i}, C \varepsilon_{n}\right)$, and let $N_{n}=\max \left\{N_{i, n}\right\}_{i=1}^{n}$. Then, as $|j-i|$ is bounded by the number of points distinct from $w_{i}$, one of the sample points, in the ball $B\left(w_{i}, C \varepsilon_{n}\right)$, we have $|j-i| \leq N_{n}$.

Now, $N_{i, n}$ is $\operatorname{Binomial}(n-1, p)$ where $p=v\left(B\left(X_{i}, C \varepsilon_{n}\right)\right)$. For $k \geq 1$, we have

$$
\mathbb{P}\left(N_{i, n} \geq k\right) \leq\left(\begin{array}{c}
n-1 \\
k
\end{array}\right) p^{k} \leq \frac{(n p)^{k}}{k !} .
$$

Recalling that $v=\rho d x$ and $\rho$ is bounded, we have $p \leq\|\rho\|_{\infty} \operatorname{Vol}(B(0,1)) \varepsilon_{n}^{d}$, and so $P\left(N_{i, n} \geq k\right) \leq\left(C^{\prime} n \varepsilon_{n}^{d}\right)^{k} / k$ ! for some constant $C^{\prime}$.

Let $N_{n}=\max \left\{N_{i, n}\right\}_{i=1}^{n}$. Then a union bound gives that

$$
P\left(N_{n} \geq k\right) \leq \frac{n}{k !} \exp \left\{k\left(\log n+d \log \varepsilon_{n}+\log C^{\prime}\right)\right\} .
$$


Taking $k=\left\lceil\varepsilon_{n}^{-\beta}\right\rceil$, and noting $k ! \geq \sqrt{2 \pi} e^{-k} k^{k+1 / 2}$, yields that

$$
P\left(N_{n} \geq \varepsilon_{n}^{-\beta}\right) \leq \frac{n}{\sqrt{2 \pi}} \exp \left\{\varepsilon_{n}^{-\beta}\left(\log n+(\beta+d) \log \varepsilon_{n}+\log C^{\prime}+1\right)\right\} .
$$

If $\varepsilon_{n}$ is in the form $\varepsilon_{n}=n^{-\delta}$, then the right-hand side of (4.10) is summable when $(\beta+d) \delta>1$.

Hence, by the Borel-Cantelli lemma, for realizations in the intersection of a probability 1 set and $A_{\mathrm{NN}}$ say, we have $\Theta_{n} \leq N_{n} \leq \varepsilon_{n}^{-\beta}$ for all large $n$, and (4.9) follows.

We now give a lower bound on the cost of certain "long necks," that is the cost of an optimal $H_{n}$-path $\mathbf{w}$ of moving away from two close by vertices.

LEMMA 4.8. Suppose $d \geq 2, p=1$ and that $f$ also satisfies (TrIneq), and (Pythag) with $\alpha>1$. Fix a realization $\left\{X_{i}\right\}$ in $A_{\mathrm{NN}}$. Suppose $\mathbf{w} \in \arg \min H_{n}$ is an optimal path, and let $i<j$ be indices such that $\left|w_{i}-w_{j}\right|<\varepsilon_{n}$. Let $\ell$ denote the straight line segment from $w_{i}$ to $w_{j}$. Consider a neighborhood $\mathcal{A}=\bigcup_{x \in \ell} B(x, r)$ of $\ell$, with $r=\varepsilon_{n}^{\alpha}$.

Then, if there is a point $w_{k} \notin \mathcal{A}$ for some $i<k<j$, there is a constant $C=$ $C(\alpha, f)$ such that

$$
\sum_{q=i}^{j-1} f\left(w_{i}, w_{q+1}-w_{q}\right) \geq f\left(w_{i}, w_{j}-w_{i}\right)+C r^{\alpha} .
$$

Suppose now $l_{\mathbf{v}} \in \arg \min L_{n}$. Then (4.11) holds with $\mathbf{v}$ in place of $\mathbf{w}$.

PROOF. The argument for $\mathbf{v}$ is the same as for $\mathbf{w}$, which we now present. Suppose a point $w_{k}$ is at least an Euclidean distance $r$ from $\ell$. By the (TrIneq) condition, $\sum_{q=i}^{j-1} f\left(w_{i}, w_{q+1}-w_{q}\right) \geq f\left(w_{i}, w_{k}-w_{i}\right)+f\left(w_{i}, w_{j}-w_{k}\right)$.

By Lemma 4.7, as $\left|w_{i}-w_{j}\right|<\varepsilon_{n}$, we have $\left|w_{k}-w_{i}\right| \leq 2\left(m_{2} / m_{1}\right) \varepsilon_{n}$, which is strictly less than an $\eta<1$ for all large $n$. We also conclude $\left|w_{j}-w_{k}\right|, \mid w_{i}-$ $w_{j} \mid<\eta<1$ for all large $n$. In addition, $2\left(m_{2} / m_{1}\right) r^{1 / \alpha}=2\left(m_{2} / m_{1}\right) \varepsilon_{n} \geq \mid w_{i}-$ $w_{j} \mid$. Thus, by (Pythag) with $x=w_{i}$, we obtain $f\left(w_{i}, w_{k}-w_{i}\right)+f\left(w_{i}, w_{j}-\right.$ $\left.w_{k}\right) \geq f\left(w_{i}, w_{j}-w_{i}\right)+C r^{\alpha}$, where $C=C(\alpha, f)$.

Hence, (4.11) follows by combining the inequalities.

We now give the proof of Lemma 4.5. The argument is in two steps. In the first step, using a rough count on the number of vertices of the path within a given box, we may approximate the contribution to $H_{n}$ and $L_{n}$ from the vertices in the box in terms of a localized cost. Then we use (Pythag), applied to the localized cost, to deduce that the optimal path in the box is trapped in a small set in the box. The second step then is to show that such small sets contain only a constant number of points in $\left\{X_{i}\right\}$. 
ProOF OF LEMma 4.5. We will give the main argument for $\mathbf{w}$ and indicate modifications with respect to v. Consider a box $\square:=\square_{n, z}$. Boxes with at most one point trivially satisfy the claim in the lemma if say $K \geq 2$. Suppose now that there are at least two points in the box.

Step 1. Let $w_{i}$ and $w_{j}$ be the first and last points of $\mathbf{w}$ in the box, that is, with the smallest and largest indices respectively. By Lemma 4.7, as $\mid w_{i}-$ $w_{j} \mid<\varepsilon_{n}$, we have $w_{k} \in B\left(w_{i}, C^{\prime} \varepsilon_{n}\right)$ for $i \leq k \leq j$. Hence, by (Lip), we have $\left|\sum_{k=i}^{j-1} f\left(w_{k}, w_{k+1}-w_{k}\right)-\sum_{k=i}^{j-1} f\left(w_{i}, w_{k+1}-w_{k}\right)\right| \leq C^{\prime} \varepsilon_{n}^{2}|j-i|$. Now, also by Lemma 4.7, when $\delta>(\beta+d)^{-1}$ for $\beta>0$, we have $|j-i| \leq \varepsilon_{n}^{-\beta}$. Hence, the following estimate, with respect to a localized energy, where $x=w_{i}$ is fixed, is obtained:

$$
\left|\sum_{k=i}^{j-1} f\left(w_{k}, w_{k+1}-w_{k}\right)-\sum_{k=i}^{j-1} f\left(w_{i}, w_{k+1}-w_{k}\right)\right| \leq C^{\prime} \varepsilon_{n}^{2-\beta} .
$$

Similarly, when $\mathbf{v}$ is considered, by the same reasoning using Lemma 4.7 and (Lip), we may obtain (4.12) with $\mathbf{v}$ in place of $\mathbf{w}$, and moreover,

$$
\begin{aligned}
& \left|\sum_{k=i}^{j-1} \int_{0}^{1} f\left(l_{v_{k}, v_{k+1}}(t), v_{k+1}-v_{k}\right) d t-\sum_{k=i}^{j-1} f\left(v_{k}, v_{k+1}-v_{k}\right)\right| \\
& \quad \leq C^{\prime} \varepsilon_{n}^{2}|j-i| \leq C^{\prime} \varepsilon^{2-\beta}
\end{aligned}
$$

Hence, combining these two estimates, we obtain that

$$
\left|\sum_{k=i}^{j-1} \int_{0}^{1} f\left(l_{v_{k}, v_{k+1}}(t), v_{k+1}-v_{k}\right) d t-\sum_{k=i}^{j-1} f\left(v_{i}, v_{k+1}-v_{k}\right)\right| \leq 2 C^{\prime} \varepsilon_{n}^{2-\beta} .
$$

Returning to $\mathbf{w}$, by Lemma 4.8 , noting $(4.12)$, any path $\left(w_{i}, \ldots, w_{j}\right)$ exiting $\mathcal{A}$, the $r=\varepsilon_{n}^{\alpha}$-neighborhood of the line segment from $w_{i}$ to $w_{j}$, is costlier, with respect to $H_{n}$, than the $H_{n}$-cost $f\left(w_{i}, w_{j}-w_{i}\right)$ of a straight path connecting $w_{i}$ to $w_{j}$ in a single hop, as follows:

$$
\begin{aligned}
\sum_{q=i}^{j-1} f\left(w_{q}, w_{q+1}-w_{q}\right)-f\left(w_{i}, w_{j}-w_{i}\right) & \geq C r^{\alpha}-C^{\prime} \varepsilon_{n}^{2-\beta} \\
& =C \varepsilon_{n}^{\alpha^{2}}-C^{\prime} \varepsilon_{n}^{2-\beta} .
\end{aligned}
$$

Let us now consider $\mathbf{v}$. Since $\left|v_{i}-v_{j}\right| \leq C^{\prime} \varepsilon_{n}$ by Lemma 4.7, using (Lip), we have that

$$
\left|f\left(v_{i}, v_{j}-v_{i}\right)-\int_{0}^{1} f\left(l_{v_{i}, v_{j}}(t), v_{j}-v_{i}\right) d t\right| \leq C^{\prime} \varepsilon_{n}^{2} .
$$

Following the same reasoning given with respect to $\mathbf{w}$, we may obtain (4.14) with $\mathbf{v}$ in place of $\mathbf{w}$. Then, noting (4.13), a path $\left(v_{i}, \ldots, v_{j}\right)$, exiting the $r=\varepsilon_{n}^{\alpha}$ neighborhood of the line segment from $v_{i}$ to $v_{j}$, has $L_{n}$ cost more than the one 
step $H_{n}$ cost $f\left(v_{i}, v_{j}-v_{i}\right)$ by the amount $C r^{\alpha}-2 C^{\prime} \varepsilon_{n}^{2-\beta}$. By (4.15), this $H_{n}$ cost differs from the one step $L_{n} \operatorname{cost} \int_{0}^{1} f\left(l_{v_{i}, v_{j}}(t), v_{j}-v_{i}\right) d t$ of moving from $v_{i}$ to $v_{j}$ by $C^{\prime} \varepsilon_{n}^{2}$.

Therefore, the cost savings of moving in one step, in considering $\mathbf{w}$ or $\mathbf{v}$ which exit the $r$-neighborhood, is bounded below by $C r^{\alpha}-3 C^{\prime} \varepsilon_{n}^{2-\beta}=O\left(\varepsilon_{n}^{\alpha^{2}}-\varepsilon_{n}^{2-\beta}\right)$, which is positive, for all large $n$, when $\alpha^{2}<2-\beta$. This is the case when we fix $\beta=\left(2-\alpha^{2}\right) \eta>0$, for an $0<\eta<1$, since $1<\alpha<\sqrt{2}$.

Hence, with this choice of $\beta$, such exiting paths are not optimal, and all the points $\left\{w_{i}, \ldots, w_{j}\right\}$ or $\left\{v_{i}, \ldots, v_{j}\right\}$ in the box must belong to the $r=\varepsilon_{n}^{\alpha}$ neighborhood of the line segment connecting the $i$ th and $j$ th points.

We note, given the value of $\beta$, to use Lemma 4.7 above, the exponent $\delta$ should satisfy $\delta>\left[\left(2-\alpha^{2}\right) \eta+d\right]^{-1}$, afforded by our assumption (2.12).

Step 2. We now focus on $\mathbf{w}$ as the following counting argument is the same with respect to $\mathbf{v}$. We will count the points in the small set $\mathcal{A}$.

To this end, given two samples $X_{i}$ and $X_{j}$, consider the line segment connecting them. Let $\ell_{i, j}$ be that portion of the line segment within distance $\varepsilon_{n}$ of $X_{i}$. Define $N_{n, i, j}$ as the number of distinct points in the $r=\varepsilon_{n}^{\alpha}$ neighborhood of $\ell_{i, j}$. Then $N_{n, i, j}$ is $\operatorname{Binomial}(n-2, p)$ where $p$ is the $v$ measure of the neighborhood. As the neighborhood is nearly covered by a cylinder with length $\varepsilon_{n}$ and radius $\varepsilon_{n}^{\alpha}$, and the density $\rho$ is bounded, $p \leq C(\rho) \varepsilon_{n}^{\alpha(d-1)+1}$. Let also $N_{n}$ be the maximum of such counts $N_{n, i, j}$ over the sample points.

Now, by Step 1 and the above definitions, the cardinality $\left|\left\{w_{k}\right\}_{k=i}^{j}\right|=|j-i|=$ : $M_{z}$ of points in the path in $\square_{z}$ is bounded by $\left|\mathcal{X}_{n} \cap \mathcal{A}\right| \leq 2+N_{n}$, which does not depend on $z$. Observe that

$$
P\left(N_{n, i, j} \geq K^{\prime}\right) \leq\left(n^{K^{\prime}} / K^{\prime} !\right) v(\mathcal{A})^{K^{\prime}} \leq C(\rho)^{K^{\prime}} n^{K^{\prime}} \varepsilon_{n}^{K^{\prime} \alpha d+K^{\prime}(1-\alpha)} .
$$

Hence, by a union of events bound, as the total number of pairs $\left(X_{i}, X_{j}\right)$ is $n(n-1)$ in the sample, we have

$$
P\left(N_{n} \geq K^{\prime}\right) \leq n^{2} \times C(\rho)^{K^{\prime}} n^{K^{\prime}} \varepsilon_{n}^{K^{\prime} \alpha d+K^{\prime}(1-\alpha)} .
$$

Therefore, we have that

$$
P\left(\max _{z} M_{z} \geq K^{\prime}+2\right) \leq P\left(N_{n} \geq K^{\prime}\right) \leq C(\rho)^{K^{\prime}} n^{K^{\prime}+2} \varepsilon_{n}^{K^{\prime} \alpha d+K^{\prime}(1-\alpha)} .
$$

Suppose $\varepsilon_{n}$ is of the form $\varepsilon_{n}=n^{-\delta}$ for $0<\delta<1 / d$. If $d<K^{\prime}<\infty$ and

$$
\delta>\left(K^{\prime}+3\right) /\left[K^{\prime}(\alpha(d-1)+1)\right],
$$

the display (4.16) is summable in $n$. In particular, when $\delta>[\alpha(d-1)+1]^{-1}$, part of our assumptions (2.12), a large but fixed $K^{\prime}$ can be chosen so that (4.17) holds.

Hence, by Borel-Cantelli lemma, on the intersection of a probability 1 set and $A_{\mathrm{NN}}$ say, we recover the claim for all large $n$ that the path visits at most $K=K^{\prime}+2$ points between the first and last visit to a visited box. 
4.2.2. Convergence of optimal paths. We now consider the behavior of the optimal paths, in analogy to Theorem 2.2, for the energy $H_{n}$.

Proposition 4.9. Consider the assumptions for the second parts of Theorems 2.5 and 2.6. Consider a discrete path $\mathbf{w}^{(n)} \in \arg \min H_{n}$ and its linear interpolation, $l_{\mathbf{w}^{(n)}}$. Then, with respect to realizations in a probability 1 subset of $A_{\mathrm{NN}} \cap A_{Q}$, for any subsequence of $\left\{l_{\mathbf{w}^{(n)}}\right\}$, and correspondingly of $\left\{\mathbf{w}^{(n)}\right\}$, there is a further subsequence of the linear paths which converges uniformly to a limit path $\gamma \in \arg \min F$, and of the discrete paths in the Hausdorff sense to $S_{\gamma}$.

If $F$ has a unique (up to reparametrization) minimizer $\gamma$, then the whole sequence $\left\{\mathbf{w}^{(n)}\right\}$ converges, $\lim _{n \rightarrow \infty} d_{\text {haus }}\left(\mathbf{w}^{(n)}, S_{\gamma}\right)=0$.

Consider now a path $l_{\mathbf{v}}(n) \in \arg \min L_{n}$. The same conclusions holds for $\left\{\mathbf{v}^{(n)}\right\}$ in place of $\left\{\mathbf{w}^{(n)}\right\}$.

Proof. Consider first $\mathbf{w}^{(n)} \in \arg \min H_{n}$. By the compactness criterion, Proposition 4.6, almost surely, any subsequence of the paths $\left\{l_{\mathbf{w}^{(n)}}\right\}$ has a further subsequence $\left\{l_{\mathbf{w}^{\left(n_{k}\right)}}\right\}$ converging uniformly to a limit $\gamma$. By the liminf Lemma 3.2, $F(\gamma) \leq \liminf _{k \rightarrow \infty} F\left(l_{\mathbf{w}^{\left(n_{k}\right)}}\right)$.

The same argument and conclusion holds with $l_{\mathbf{v}^{(n)}} \in \arg \min L_{n}$ and $\mathbf{v}^{(n)}$ in place of $l_{\mathbf{w}^{(n)}}$ and $\mathbf{w}^{(n)}$.

We now show that $\gamma \in \arg \min F$. With respect to optimal $L_{n}$ paths, as $F\left(l_{\mathbf{v}^{(n)}}\right)=\min L_{n}$, and $\min L_{n} \rightarrow \min F$ a.s. by Proposition 4.3 , we obtain $F(\gamma) \leq$ $\min F$, and so the desired conclusion.

For $H_{n}$ optimal paths, we recall an argument in the proof of Theorem 2.4. Using only the standing assumptions (allowing $p=1$ ) and (Lip), we derived (4.2), namely, for $\mathbf{u} \in V_{n}(a, b)$, that

$$
\left|L_{n}\left(l_{\mathbf{u}}\right)-H_{n}(\mathbf{u})\right| \leq c m_{1}^{-1} \varepsilon_{n} \min \left(L_{n}\left(l_{\mathbf{u}}\right), H_{n}(\mathbf{u})\right),
$$

where $c$ is the constant in (Lip). Then, as a consequence of Proposition 4.1, we saw in (4.4) that $\min L_{n} \leq L_{n}\left(l_{\mathbf{w}^{(n)}}\right) \leq\left(\mathrm{cm}_{1}^{-1} \varepsilon_{n}+1\right)^{2} \min L_{n}$. Since, by Proposition $4.3, \min L_{n} \rightarrow \min F$ a.s., we conclude that $\gamma \in \arg \min F$.

Finally, we remark that the Hausdorff convergences are argued as in the proof of Corollary 2.3.

\section{APPENDIX}

Here, we collect some results which we had previously assumed.

\section{A.1. Nearest-neighbor rate.}

Proposition A.1. Let $\left\{X_{i}\right\}$ be i.i.d. samples from a probability measure $v=$ $\rho(x) d x$ on a Lipschitz domain $D$, and let

$$
R_{n}=\sup _{y \in D} \min _{1 \leq i \leq n}\left|X_{i}-y\right|
$$


Suppose $\rho(x)$ is uniformly bounded below by a positive constant. Then there exists a constant $C$, independent of $n$, such that, for almost all realizations $\left\{X_{i}\right\}$,

$$
\limsup _{n \rightarrow \infty} \frac{R_{n} n^{1 / d}}{(\log n)^{1 / d}} \leq C .
$$

In particular, when $\varepsilon_{n}$ satisfies (2.6), for $a, b \in D$, almost surely for all large $n$, the graph $\mathcal{G}_{n}(a, b)$ is connected.

PROOF. We first address the claim with respect to $R_{n}$. Let $B(y, r)$ be the Euclidean ball of radius $r$ centered at $y \in D$. Since $D$ is Lipschitz, there is a constant $c$ such that $m(B(y, r) \cap D) \geq c m(B(y, r))$ for all small $r>0$, where $m$ denotes Lebesgue measure (cf. the discussion about cone conditions in Section 4.11 of [1]). It follows that there is a constant $c$ such that $m(B(y, r) \cap D) / m(D) \geq c r^{d}$ for all $y \in D$ and all small $r>0$. Since $v$ has density $\rho$ bounded below by a positive constant, there exists a constant $c$ such that $v(B(y, r)) \geq c r^{d}$ for all $y \in D$ and $0<r<r_{0}$, where $r_{0}$ is a sufficiently small constant. Therefore, recalling $\mathcal{X}_{n}=\left\{X_{1}, X_{2}, \ldots, X_{n}\right\}$, we have

$$
\begin{aligned}
\mathbb{P}\left(\left|B(y, r) \cap \mathcal{X}_{n}\right|=0\right) & =(1-v(B(y, r)))^{n} \\
& \leq\left(1-c r^{d}\right)^{n} \leq e^{-c n r^{d}} .
\end{aligned}
$$

Let $\left\{y_{1}, \ldots, y_{k}\right\} \subset D$ be a collection of points so that $\sup _{x \in X} \min _{1 \leq i \leq k}\left|x-y_{i}\right| \leq$ $r$. We may take the number of points $k$ to satisfy $k \leq c / r^{d}$ for some constant $c$ independent of $r$, say, by choosing $\left\{y_{i}\right\}$ to be a regular grid, with grid length $\sim r$.

Let $E_{i}$ denote the event that $\left|B\left(y_{i}, r\right) \cap \mathcal{X}_{n}\right|=0$, and consider the event $\left\{R_{n}>\right.$ $2 r\}$ that there exists a $y \in D$ with $\min _{1 \leq i \leq n}\left|X_{i}-y\right|>2 r$. Then, by a triangle inequality argument, we have $\left\{R_{n}>2 r\right\} \subset \bigcup_{i=1}^{k} E_{i}$. Hence, together with (A.1), we have

$$
\mathbb{P}\left(R_{n}>2 r\right) \leq \sum_{i=1}^{k} \mathbb{P}\left(E_{i}\right) \leq \frac{c}{r^{d}} e^{-c n r^{d}} .
$$

Let $r^{d}=(3 \log n) /(c n)$. Then (A.2) gives a summable term,

$$
\mathbb{P}\left(R_{n}>2 \frac{(3 \log n)^{1 / d}}{n^{1 / d}}\right) \leq \frac{c^{2}}{3 n^{2}} .
$$

By the Borel-Cantelli lemma, $R_{n} \leq 2(3 \log n)^{1 / d} / n^{1 / d}$ for all large $n$.

We now show that $\mathcal{G}_{n}(a, b)$ is connected when $\varepsilon_{n}$ satisfies (2.6). Let $v_{1}, v_{2}$ be any vertices in $\mathcal{X}_{n} \cup\{a, b\}$, and consider the line $\ell(t)=v_{1}(1-t)+v_{2}(t)$ between them for $t \in[0,1]$. By convexity of $D$, the path $\ell$ is contained in $D$. Consider points on the path $v_{1}=\ell(0), \ell\left(R_{n}\right), \ell\left(2 R_{n}\right), \ldots, \ell\left(k R_{n}\right), \ell(1)=v_{1}$, where $k=$ $\left\lfloor\left|v_{2}-v_{1}\right| / R_{n}\right\rfloor$ so that $\left|1-k R_{n}\right| \leq R_{n}$. Each point $y=\ell\left(j R_{n}\right)$ is within Euclidean distance $R_{n}$ of a point $u_{j} \in \mathcal{X}_{n}$. By construction, for $1 \leq j \leq k-1$, the Euclidean 
distance between $u_{j}$ and $u_{j+1}$ is less than sum of the distances, from $u_{j}$ to $\ell\left(j R_{n}\right)$, from $\ell\left(j R_{n}\right)$ to $\ell\left((j+1) R_{n}\right)$, and from $\ell\left((j+1) R_{n}\right)$ to $u_{j+1}$, which is bounded by $3 R_{n}$. Similarly, the endpoints $v_{1}, v_{2}$ are within Euclidean distance $2 R_{n}$ of $u_{1}$ and $u_{k}$, respectively. Since, by $(2.6), 3 R_{n} / \varepsilon_{n}<1$ for all large $n$, the path along vertices $\left\{v_{1}, u_{1}, \ldots, u_{k}, v_{2}\right\}$ belongs to $V_{n}(a, b)$ and so $v_{1}$ and $v_{2}$ are connected in $G_{n}(a, b)$ a.s. for all large $n$.

A.2. Existence of quasinormal minimizers. We discuss a conservation law for $F$-minimizing paths, and existence of $F$-minimizing Lipschitz paths, following the treatment in [9].

Proposition A.2. Consider the integral functional $F(\gamma)=\int_{0}^{1} f(\gamma, \dot{\gamma}) d t$, where $f$ satisfies (A0)-(A3). Then, $F$ attains a minimum on the set $\Omega(a, b)$ of Lipschitz paths from $a$ to $b$. In other words, there exists $a \gamma^{*} \in \Omega(a, b)$ with $F\left(\gamma^{*}\right)=\inf _{\gamma \in \Omega(a, b)} F(\gamma)$.

In the case that $p=1$, there exists a $\gamma \in \arg \min F$ and constants $c, c_{1}, c_{2}$ such that

$$
f(\gamma(t), \dot{\gamma}(t))=c \quad \text { for a.e. } t \in[0,1]
$$

and

$$
c_{1} \leq|\dot{\gamma}(t)| \leq c_{2} \quad \text { for a.e. } t \in[0,1] .
$$

If $p>1$, then there are constants $c, c_{1}, c_{2}$ such that (A.3) and (A.4) hold for any $\gamma \in \arg \min F$.

PROOF. We first give an argument in the case where $p>1$. Note, by assumption, the integrand $f$ is continuous and $C^{1}$ on $D \times\left(\mathbb{R}^{d} \backslash\{0\}\right)$, convex and $p$ homogenous in the second argument, and satisfies (2.4). As $p>1, f$ may be extended continuously to a $C^{1}$ function on $D \times \mathbb{R}^{d}$.

When the domain of $F$ is extended to all Sobolev paths $\gamma \in W^{1, p}([0,1] ; D)$ with $\gamma(0)=a, \gamma(1)=b$, the existence of a minimizer follows from Remark 2 of Section 3.2 in [9]. In particular, the continuity and convexity assumptions (A0) and (A1) imply that $F$ is lower-semicontinuous with respect to weak convergence of Sobolev functions. The existence of a minimizer then follows from a standard compactness argument.

Let $\gamma$ denote such a Sobolev minimizer. Consider now an inner variation $\omega(t, \varepsilon)=\gamma(\xi(t, \varepsilon))$ of $\gamma$, where $\xi$ is $C^{1}$ on $[0,1] \times\left(-\varepsilon_{0}, \varepsilon_{0}\right)$ for some $\varepsilon_{0}>0$ and $\xi(\cdot, \varepsilon)$ is a $C^{1}$ diffeomorphism of the interval $[0,1]$ to itself, with $\gamma(t, 0)=t$. It may be shown (see the discussion on pages 19-21, Proposition 1.16 and Remark 3 in Section 1.1 of [9]) that the optimality condition $\left.\frac{d}{d \varepsilon} F(\omega(\cdot, \varepsilon))\right|_{\varepsilon=0}$ over the class of inner variations, together with Euler's identity for homogenous functions, $v \cdot \nabla_{v} f(x, v)=p f(x, v)$, together imply, for some constant $c$, that

$$
(p-1) f(\gamma(t), \dot{\gamma}(t))=c \quad \text { for a.e. } t \text {. }
$$


Finally, by assumption (A3) on $f$, it follows that $c>0$ and there exist constants $c_{1}, c_{2}>0$ with $c_{1} \leq|\dot{\gamma}(t)| \leq c_{2}$ for almost every $t$. In particular, $\gamma \in \Omega(a, b)$, and the proposition is proved for $p>1$.

The argument for the $p=1$ case is complicated by a lack of compactness with respect to weak convergence in the Sobolev space $W^{1,1}([0,1] ; D)$, as well as difficulty in establishing an analogue of (A.5). By a more involved argument, relating optimizers of $F$ to optimizers of the quadratic functional $Q(\gamma):=\int_{0}^{1} f^{2}(\gamma, \dot{\gamma}) d t$, the existence of a Lipschitz path $\gamma \in \arg \min F$ satisfying (A.3) is established in Theorem 1 of [16] (see also Theorem 5.22 of [9] which gives an alternative argument). From this and assumption (A3), inequality (A.4) follows.

Acknowledgments. We would like to thank the referees for their careful and constructive comments.

\section{REFERENCES}

[1] Adams, R. A. and Fournier, J. J. F. (2003). Sobolev Spaces, 2nd ed. Pure and Applied Mathematics (Amsterdam) 140. Elsevier/Academic Press, Amsterdam. MR2424078

[2] Agranovich, M. S. (2015). Sobolev Spaces, Their Generalizations and Elliptic Problems in Smooth and Lipschitz Domains. Springer, Cham. MR3287270

[3] Alamgir, M. and von LuXburg, U. (2012). Shortest path distance in random $k$ nearest neighbor graphs. In Proc. 29th Int. Conf. on Machine Learning. Available at arXiv:1206.6381.

[4] Álvarez Paiva, J. C. (2006). Some problems on Finsler geometry. In Handbook of Differential Geometry. Vol. II 1-33. Elsevier/North-Holland, Amsterdam. MR2194667

[5] Auffinger, A., Damron, M. and Hanson, J. (2017). 50 Years of First-Passage Percolation. University Lecture Series 68. Amer. Math. Soc., Providence, RI. MR3729447

[6] BERn, M. et al. (1999). Emerging Challenges in Computational Topology. NSF report. Available at arXiv:cs/9909001.

[7] Bernstein, M., De Silva, V., Langford, J. and Tenenbaum, J. (2000). Graph approximations to geodesics on embedded manifolds. Technical Report, Dept. Psychology, Stanford Univ., Stanford, CA.

[8] Burago, D., Burago, Y. and Ivanov, S. (2001). A Course in Metric Geometry. Graduate Studies in Mathematics 33. Amer. Math. Soc., Providence, RI. MR1835418

[9] Buttazzo, G., Giaquinta, M. and Hildebrandt, S. (1998). One-Dimensional Variational Problems: An Introduction. Oxford Lecture Series in Mathematics and Its Applications 15. Oxford Univ. Press, Oxford. MR1694383

[10] CABello, S. and JEJCIC, M. (2015). Shortest paths in intersection graphs of unit disks. Comput. Geom. 48 360-367. MR3296073

[11] Crane, K., Weischedel, C. and Wardetzky, M. (2013). Geodesics in heat: A new approach to computing distance based on heat flow. ACM Trans. Graph. 28106.

[12] Garcia Trillos, N. (2017). Gromov-Hausdorff limit of Wasserstein spaces on point clouds. Available at arXiv:1702.03464.

[13] Gelfand, I. M. and Smirnov, M. M. (1994). Lagrangians satisfying Crofton formulas, Radon transforms, and nonlocal differentials. Adv. Math. 109 188-227. MR1304752

[14] Giesen, J. and Wagner, U. (2004). Shape dimension and intrinsic metric from samples of manifolds. Discrete Comput. Geom. 32 245-267. MR2074340 
[15] Hashimoto, T. B., Sun, Y. and JaAkkola, T. S. (2015). Metric recovery from directed unweighted graphs. In Proc. 18th Int. Conference on AI and Stat (AISTATS) 38, JMLR: W\&CP, San Diego, CA.

[16] Hildebrandt, S. (2015). Minimizers of one-dimensional parametric variational integrals. St. Petersburg Math. J. 27 569-576.

[17] Hirsch, C., Neuhäuser, D., Gloaguen, C. and Schmidt, V. (2015). First passage percolation on random geometric graphs and an application to shortest-path trees. $A d v$. in Appl. Probab. 47 328-354. MR3360380

[18] Howard, C. D. and Newman, C. M. (1997). Euclidean models of first-passage percolation. Probab. Theory Related Fields 108 153-170. MR1452554

[19] Howard, C. D. and Newman, C. M. (2001). Geodesics and spanning trees for Euclidean first-passage percolation. Ann. Probab. 29 577-623. MR1849171

[20] Hwang, S. J., Damelin, S. B. and Hero, A. O. III (2016). Shortest path through random points. Ann. Appl. Probab. 26 2791-2823. MR3563194

[21] LaGatta, T. and WeHR, J. (2010). A shape theorem for Riemannian first-passage percolation. J. Math. Phys. 51 053502, 14. MR2666980

[22] LaGatta, T. and WeHR, J. (2014). Geodesics of random Riemannian metrics. Comm. Math. Phys. 327 181-241. MR3177936

[23] MaZ'YA, V. (2011). Sobolev Spaces with Applications to Elliptic Partial Differential Equations, 2nd ed. Grundlehren der Mathematischen Wissenschaften 342. Springer, Heidelberg. MR2777530

[24] MÉmoli, F. and SAPIRO, G. (2005). Distance functions and geodesics on submanifolds of $\mathbb{R}^{d}$ and point clouds. SIAM J. Appl. Math. 65 1227-1260. MR2147326

[25] Papadopoulos, A. (2014). Hilbert's fourth problem. In Handbook of Hilbert Geometry (A. Papadopoulos and M. Troyanov, eds.). IRMA Lect. Math. Theor. Phys. 22 391-431. Eur. Math. Soc., Zürich. MR3329889

[26] Penrose, M. (2003). Random Geometric Graphs. Oxford Studies in Probability 5. Oxford Univ. Press, Oxford. MR1986198

[27] Peyré, G., Péchaud, M., Keriven, R., Cohen, L. et al. (2010). Geodesic methods in computer vision and graphics. Foundations and Trends in Computer Graphics and Vision 5 197-397.

[28] SAJAmA and OrLitZKY, A. (2005). Estimating and computing density based distance metrics. In Proc. 22nd Int. Cont. on Machine Learning, Bonn, Germany.

[29] Sedgewick, R. and Vitter, J. S. (1986). Shortest paths in Euclidean graphs. Algorithmica 1 31-48. MR0833117

[30] Sethian, J. A. (1999). Fast marching methods. SIAM Rev. 41 199-235. MR1684542

[31] TAMÁSSY, L. (2008). Relation between metric spaces and Finsler spaces. Differential Geom. Appl. 26 483-494. MR2458274

[32] Tenenbaum, J. B., De Silva, V. and Langford, J. C. (2000). A global geometric framework for nonlinear dimensionality reduction. Science $2902319-2323$.

[33] von Deylen, S. W., Glickenstein, D. and Wardetzky, M. (2016). Distortion estimates for barycentric coordinates on Riemannian simplices. Available at arXiv:1610.01168.

[34] Yu, H., Zhang, J. J. and Jiao, Z. (2014). Geodesics on point clouds. Math. Probl. Eng. Art. ID 860136, 12. MR3206968

DEPARTMENT OF MATHEMATICS

UNIVERSITY OF ARIZONA

TUCSON, ARIZONA 85721

USA

E-MAIL: edavis@math.arizona.edu sethuram@math.arizona.edu 